\title{
Interruption of progerin-lamin A/C binding ameliorates Hutchinson-Gilford progeria syndrome phenotype
}

\author{
Su-Jin Lee, ${ }^{1}$ Youn-Sang Jung, ${ }^{1}$ Min-Ho Yoon, ${ }^{1}$ So-mi Kang, ${ }^{1}$ Ah-Young Oh, ${ }^{1}$ Jee-Hyun Lee, ${ }^{2}$ So-Young Jun, ${ }^{3}$ Tae-Gyun Woo, ${ }^{1}$ \\ Ho-Young Chun, ${ }^{1}$ Sang Kyum Kim, ${ }^{2}$ Kyu Jin Chung, ${ }^{2}$ Ho-Young Lee, ${ }^{4}$ Kyeong Lee, ${ }^{5}$ Guanghai Jin, ${ }^{5}$ Min-Kyun Na, ${ }^{2} \mathrm{Nam}$ Chul Ha, ${ }^{3}$ \\ Clea Bárcena, ${ }^{6}$ José M.P. Freije, ${ }^{6}$ Carlos López-Otín, ${ }^{6}$ Gyu Yong Song, ${ }^{2}$ and Bum-Joon Park ${ }^{1}$

\begin{abstract}
'Department of Molecular Biology, College of Natural Science, Pusan National University, Busan, South Korea. ²College of Pharmacy, Chungnam National University, Deajon, South Korea.
${ }^{3}$ College of Pharmacy, Pusan National University, Busan, South Korea. ${ }^{4}$ Department of Nuclear Medicine, College of Medicine, Seoul National University Bundang Hospital, Seongnam, South Korea. ${ }^{5}$ BK21 Plus R-FIND Team, College of Pharmacy, Dongguk University, Goyang, South Korea. Departamento de Bioquímica y Biología Molecular, Facultad de Medicina, Instituto Universitario de Oncología-IUOPA, Universidad de Oviedo, Oviedo, Spain.
\end{abstract}

\begin{abstract}
Hutchinson-Gilford progeria syndrome (HGPS) is a rare autosomal dominant genetic disease that is caused by a silent mutation of the LMNA gene encoding lamins $A$ and $C$ (lamin $A / C$ ). The $G 608 \mathrm{C}$ mutation generates a more accessible splicing donor site than does WT and produces an alternatively spliced product of LMNA called progerin, which is also expressed in normal aged cells. In this study, we determined that progerin binds directly to lamin $A / C$ and induces profound nuclear aberrations. Given this observation, we performed a random screening of a chemical library and identified 3 compounds $(\mathrm{JH} 1, \mathrm{JH} 4$, and $\mathrm{JH} 13$ ) that efficiently block progerin-lamin $\mathrm{A} / \mathrm{C}$ binding. These 3 chemicals, particularly JH4, alleviated nuclear deformation and reversed senescence markers characteristic of HCPS cells, including growth arrest and senescenceassociated $\beta$-gal (SA- $\beta$-gal) activity. We then used microarray-based analysis to demonstrate that JH4 is able to rescue defects of cell-cycle progression in both HGPS and aged cells. Furthermore, administration of JH4 to Lmna ${ }^{\mathrm{C609C/C609C}}$-mutant mice, which phenocopy human HGPS, resulted in a marked improvement of several progeria phenotypes and an extended lifespan. Together, these findings indicate that specific inhibitors with the ability to block pathological progerin-lamin A/C binding may represent a promising strategy for improving lifespan and health in both HCPS and normal aging.
\end{abstract}

\section{Introduction}

Hutchinson-Gilford progeria syndrome (HGPS) is an extremely rare disease that recapitulates some aspects of physiological aging (1-5). Accordingly, a detailed understanding of the molecular mechanisms underlying HGPS may be useful for the prevention of aging or aging-related diseases. In fact, it has been shown that progerin, a gene product of HGPS cells derived from an aberrant splicing process $(6,7)$, is also detected in non-HGPS aged cells $(8$, $9)$. Progerin expression induces several changes in cellular morphology, such as nuclear membrane blebs and a reduction of nucleoplasmic lamin A $(10,11)$, whereas progerin downregulation rescues nuclear deformation $(10,12)$, indicating that progerin gain of function is a causal factor for HGPS. It has also been reported that progerin accumulates in several human cancer cells $(13,14)$ and induces nuclear deformation (9-11). Notably, expression levels of progerin and lamins A and C (lamin A/C) are significantly reduced in induced pluripotent stem cells (iPSCs) derived from patients with HGPS $(15,16)$. Likewise, cellular senescence markers, including nuclear deformation, histon e H3 trimethyl Lys9 (H3K9Me3) and senescence-associated $\beta$-gal (SA- $\beta$-gal), are also decreased in these cells. By contrast, differentiated HGPS cells re-express senescence markers following progerin expression (15).

Authorship note: S.J. Lee and Y.S. Jung contributed equally to this work.

Conflict of interest: The authors have declared that no conflict of interest exists.

Submitted: August 10, 2015; Accepted: August 4, 2016.

Reference information: / Clin Invest. 2016;126(10):3879-3893. doi:10.1172/JCI84164.
Despite several reports dealing with the role of this protein in cell-cycle regulation $(11,17,18)$ and senescence $(12,19)$, how progerin induces various cellular defects and premature aging remains to be revealed. Remarkably, proteomic studies and 2-hybrid approaches have identified lamin $\mathrm{A} / \mathrm{C}$ as a progerin-binding protein $(20,21)$. On this basis, we hypothesized that the interaction of progerin with lamin $\mathrm{A} / \mathrm{C}$ contributes to the development of the senescence phenotype of HGPS and aged cells.

\section{Results}

Direct interaction between progerin and lamin A/C but not lamin $B$. To investigate our hypothesis, we performed a binding assay and found that $\mathrm{C}$-terminal progerin and lamin $\mathrm{A}$ associated with lamins $\mathrm{A}$ and $\mathrm{C}$ but not with lamin B1 (Figure 1A and Supplemental Figure 1A; supplemental material available online with this article; doi:10.1172/JCI84164DS1). In addition, the binding affinity between progerin and lamin $\mathrm{A} / \mathrm{C}$ was stronger than that between lamin A/C and lamin A/C (Figure 1B and Supplemental Figure 1B). To dissect the progerin domain involved in lamin A/C binding, we performed a glutathione-S-transferase pull-down (GST pull-down) assay using a His-tagged lamin A N-terminal region (LMNA-N; aa residues 1-300) and a lamin A middle region (LMNA-M; aa residues 301-564) with bead-coupled GST-C-terminal fragments of lamin A (GST-LMNA) or progerin (GSTprogerin). We found that the middle region of lamin A was responsible for progerin binding (Figure 1, C and D). We could confirm the direct interaction of both proteins through Far Western blot 
A

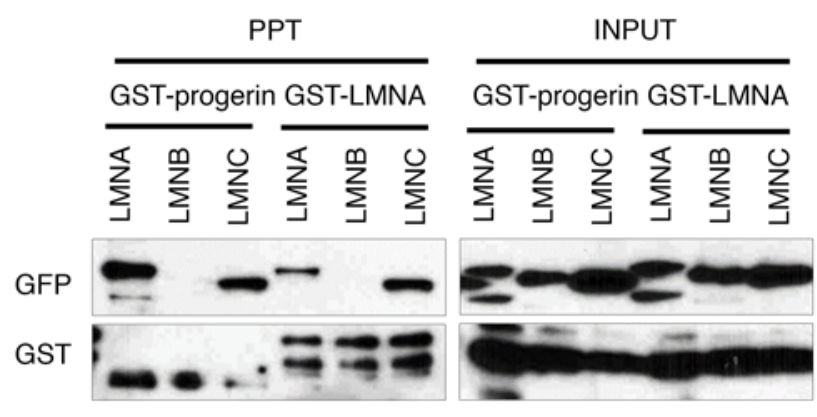

B

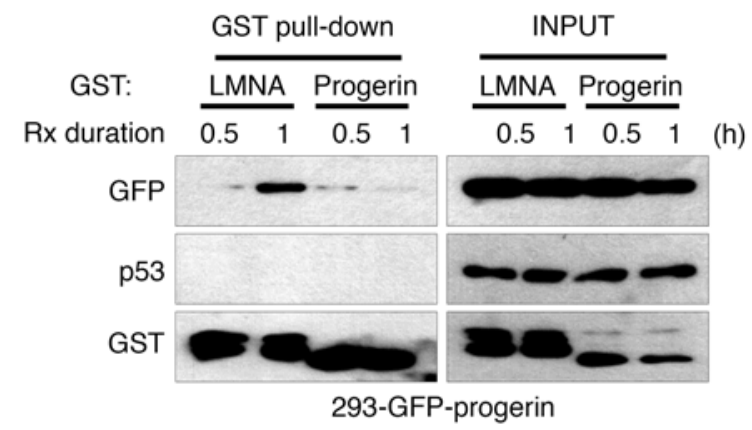

C GST:

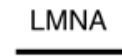

Progerin

His-LMNA $\bar{N} \quad \bar{N} \quad \bar{N} \quad M$ Input: His

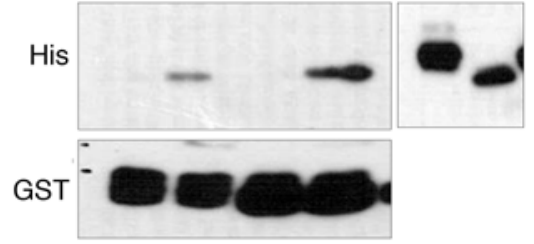

D

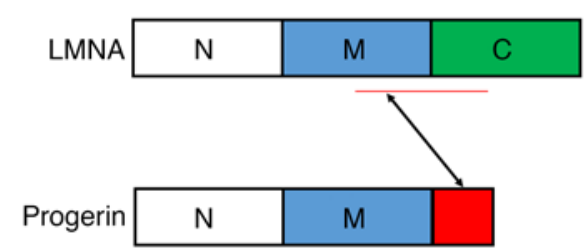

$\mathbf{F}$
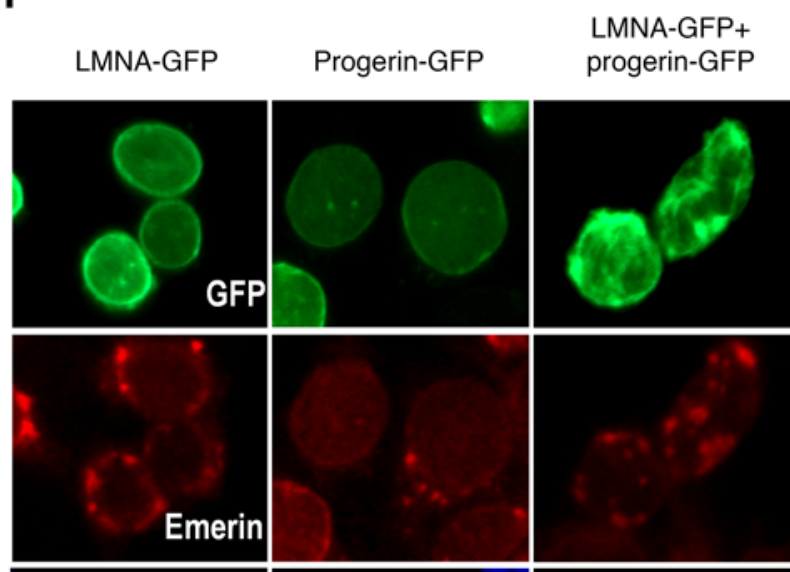

E

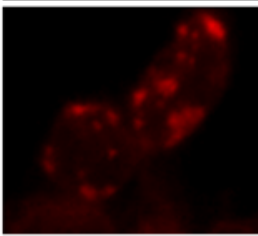

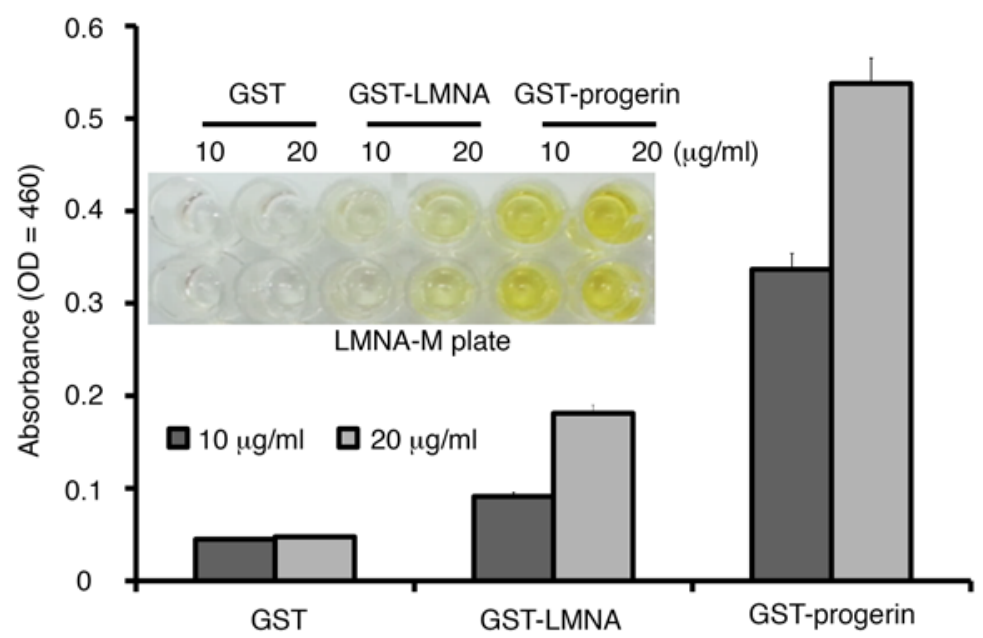

G
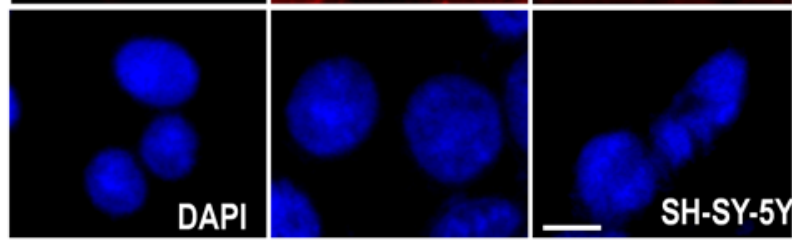

SH-SY-5Y

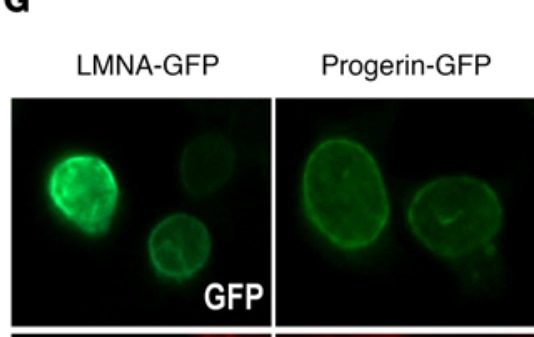

LMNA-GFP+ progerin-GFP
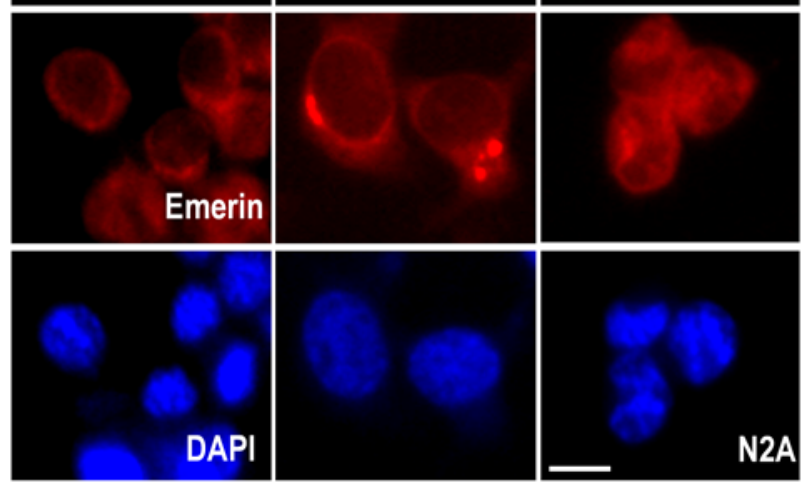
Figure 1. Identification of a lamin A-progerin-binding inhibitor. (A) Interaction of progerin and LMNA A/C but not lamin B. GST pull-down assay using lysates from HEK293 cells transfected with lamins A, B, C and progerin after incubation with the $C$-terminal 100 -aa sequence of lamin A (564-664 aa; CST-LMNA) or 50-aa sequence of progerin (564-614 aa; GST-progerin). (B) Strong interaction between lamin A and progerin. Lamin A-progerin interaction was detected after a 1-hour incubation. However, progerin did not show evidence of self-association. Rx, treatment. (C) Direct interaction between lamin $A$ and progerin. In vitro-binding assay using His-tagged N-terminal (1-300 aa; LMNA-N) and the middle region (LMNA-M) of lamin A after incubation with GST-LMNA or CST-progerin. Associated proteins were analyzed by Western blotting. N, LMNA-N; M, LMNA-M. (D) Scheme of the interaction between lamin A and progerin. (E) ELISA results. His-tagged LMNA-M was fixed in a 96-well plate and incubated with GST-LMNA or GST-progerin. Photograph of the 96-well plate (inset) and ELISA value. (F and $\mathbf{G}$ ) Progerin did not induce nuclear deformation in neuron or embryonic neuron cells. Transfection of GFP-progerin or lamin A alone into the neuroblastoma cell line SH-SY-5Y (F) and the mouse neuroembryonic cell line N2A did not induce nuclear deformation. However, cotransfection could induce it. Cells were transfected with the indicated vectors for 24 hours. For visualization of the nuclear membrane, cells were stained with emerin (red) and DAPI for DNA (blue). Scale bar: $10 \mu \mathrm{m}$.

analysis (Supplemental Figure 1C). Considering that progerin can strongly interact with LMNC (which corresponds to aa 1-572 of LMNA), we excluded the possibility that the C-terminal extension region of LMNA is a target of progerin. By ELISAs with an immobilized LMNA-M domain and the GST-fused recombinant lamin A or progerin, we could estimate that progerin-lamin A binding affinity is more than 2-fold stronger than that of lamin A-lamin A binding (Figure 1E). Considering that lamins $\mathrm{A}$ and $\mathrm{C}$ form intermediate filaments by head-to-tail binding and bundle through the $\mathrm{C}$-terminal and middle regions (22), progerin may induce a stronger or tighter but irregular bundle formation than does lamin $\mathrm{A}$ or $\mathrm{C}$ alone. This hypothesis was consistent with the HGPS cell phenotype, in which lamins A and C formed an irregular fiber arrangement (Supplemental Figure 1, D and E). Indeed, progerin alone did not induce nuclear deformation in lamin $\mathrm{A} / \mathrm{C}$-deficient neuron or embryonic neuron cells, whereas cotransfection with lamin A could induce it (Figure 1, F and G, and Supplemental Figure 1, F and G), despite low expression of progerin in N2A cells (Supplemental Figure 1G). These results indicated that nuclear lamina alteration can derive from the strong binding of progerin to lamins A and C.

Screening of specific binding inhibitor of progerin-lamin A binding. The above findings prompted us to search for putative progerin/lamin A-binding inhibitors by using an ELISA-based screening system. In brief, progerin, immobilized in 96-well plates, was incubated sequentially with a chemical library and His-lamin A (Supplemental Figure 2, A and B). This screening identified 5 chemicals that blocked this interaction (JH1, JH4, JH13, LG-02, and KEK-2-24, Supplemental Figure 2C). Because KEK-2-24 is a mixture of compounds from Panax ginseng and LG-02 requires complicated synthetic steps, we excluded them from further study. A detailed synthetic pathway for the selected chemicals is described in the Chemical Synthesis section of the Supplemental Methods. Interestingly, in spite of the blind screening, the selected chemicals were structurally similar (Supplemental Figure 2C) and showed very low cytotoxicity (Supplemental Figure 2D). Therefore, we decided to further investigate the activity of JH chemi- cals, particularly JH4, because JH1 and JH13, despite their similar effects, show very low solubility. We first confirmed their inhibitory effect of the $\mathrm{JH}$ chemicals on the binding between progerin and lamin A through in vitro binding assays (Supplemental Figure 2E). Indeed, less than $1 \mu \mathrm{M}$ of JH $4\left(\mathrm{IC}_{50}=0.65 \mu \mathrm{M}\right)$ could block $50 \%$ of the interaction between progerin and lamin $\mathrm{A}$ without disruption of lamin A self-association (Supplemental Figure 2E).

The effect of progerin-lamin A-binding inhibitors in HGPS model cells. To confirm the inhibitory effect of $\mathrm{JH}$ chemicals on binding between progerin and lamin A, we performed the GST pull-down assay and observed that JH4 blocked the interaction of GST-lamin A with GFP-progerin, but not with GFP-lamin A, in a GST pulldown assay using cell lysates (Figure 2A and Supplemental Figure 3A). However, the farnesyltransferase inhibitor FTI-277, known to ameliorate nuclear deformation $(23,24)$, did not show an obvious effect on progerin-lamin A binding (Figure 2A and Supplemental Figure $3 \mathrm{~B})$. Next, we checked their in vivo effect in progerin and GFP-lamin A-transfected HEK293 cells through IP assays with an anti-GFP Ab. The binding between progerin and lamin A was markedly reduced by the $\mathrm{JH}$ chemicals, while the binding between lamin A and lamin B was not affected by these compounds (Figure 2B). Additionally, a reduction of $\mathrm{p} 16^{\mathrm{INK} 4 \mathrm{~A}}$ expression was detected in response to treatment with the $\mathrm{JH}$ chemicals (Figure 2B). We also observed the different localization of progerin from that of lamin A upon JH4 treatment (Figure 2C and Supplemental Figure 3C). Moreover, all these $\mathrm{JH}$ compounds, and in particular JH4, blocked nuclear deformation (Supplemental Figure 4, A and B). These results indicate that JH4 (and JH13 to some extent) blocked the interaction between progerin and lamin A and alleviated progerin-induced nuclear deformation. However, JH4 did not show any significant effect on the localization pattern of other laminopathy-related lamin A mutants (2) such as nuclear speckles of D192G (Supplemental Figure 4C). This result also supports the idea that JH4 possesses selective activity on progerin. Next, we examined the effect of JH chemicals on HGPS cells. Consistent with the above results, JH chemicals, in particular JH4, blocked the interaction between progerin and lamin $\mathrm{A}$ in these progeroid cells (Figure 2D and Supplemental Figure 4D), without alteration of progerin mRNA expression (Supplemental Figure 4E). In addition, $\mathrm{JH}$ chemicals, and especially $\mathrm{JH} 4$, ameliorated the frequency of nuclear deformation of HGPS cells (Figure 2, E and F, and Supplemental Figure 4, F and G).

Specific interaction of $\mathrm{JH} 4$ and progerin. To determine the direct target of $\mathrm{JH}$ chemicals, we monitored their effect on the previously observed progerin-p14 ${ }^{\mathrm{ARF}}$ binding (14). In this assay, we found that $\mathrm{JH}$ chemicals blocked the interaction between progerin and $\mathrm{p} 14^{\mathrm{ARF}}$ (Supplemental Figure 5A), but not between p53 and p14 ${ }^{\mathrm{ARF}}$ (ref. 25 and Supplemental Figure 5B). In addition, JH4 did not block the interaction between progerin and MEL18/BMI1 that is achieved by the middle region of lamin A (ref. 26 and Supplemental Figure 5 , C-E). Considering these facts, the C-terminal region of progerin may be the direct target of JH4 (Supplemental Figure 5F). To verify this hypothesis, we generated biotinylated JH4 (Supplemental Figure 5G; B33: precursor for biotinylation; 07: biotinylated JH4; 08: biotin plus linker). Modified chemicals showed inhibitory activity similar to that of $\mathrm{JH} 4$ on binding between lamin A and progerin (Supplemental Figure 5H) as well as on nuclear deformation (Fig- 
A

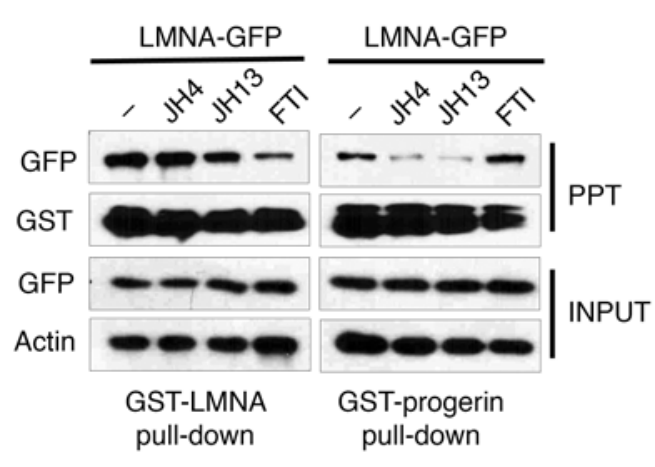

B

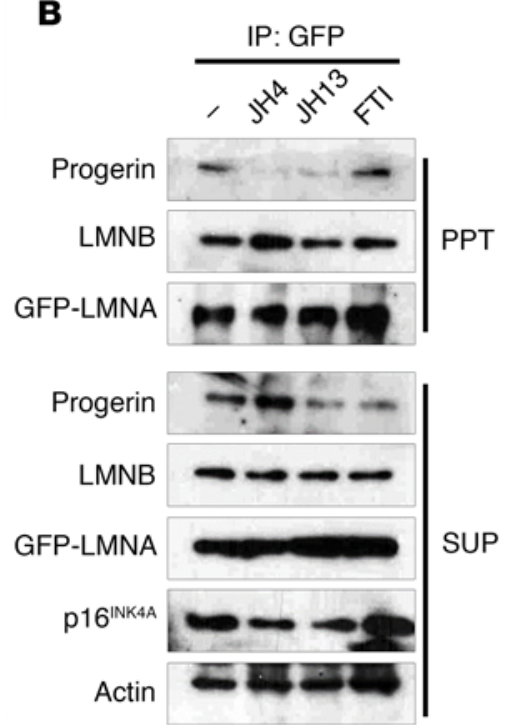

293-LMNA-GFP + NT-progerin

E

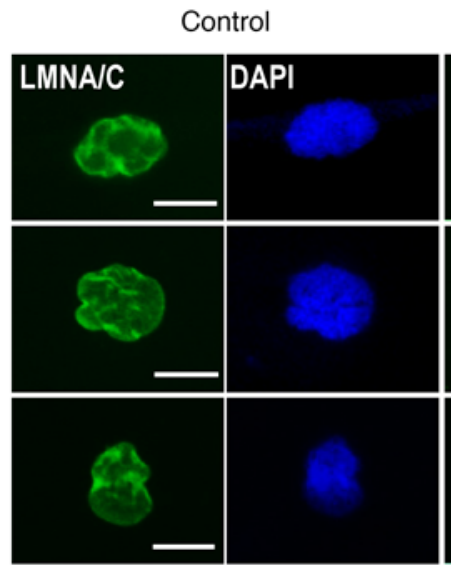

D
C

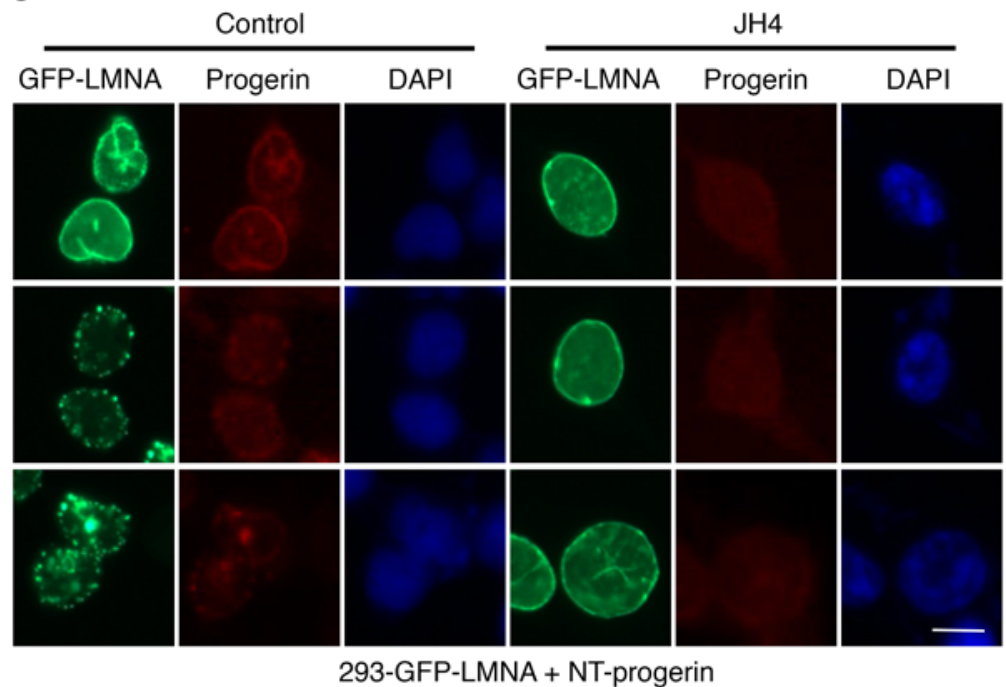

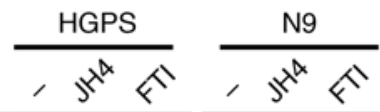

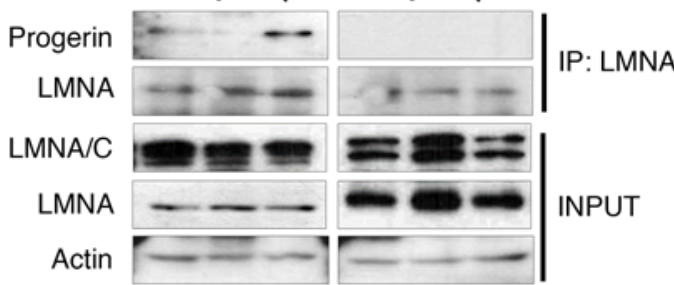

JH4

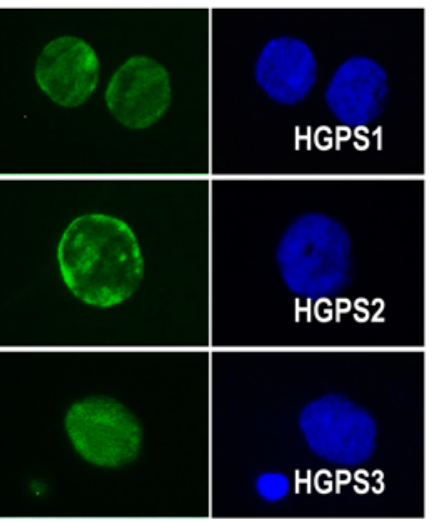

$\mathbf{F}$

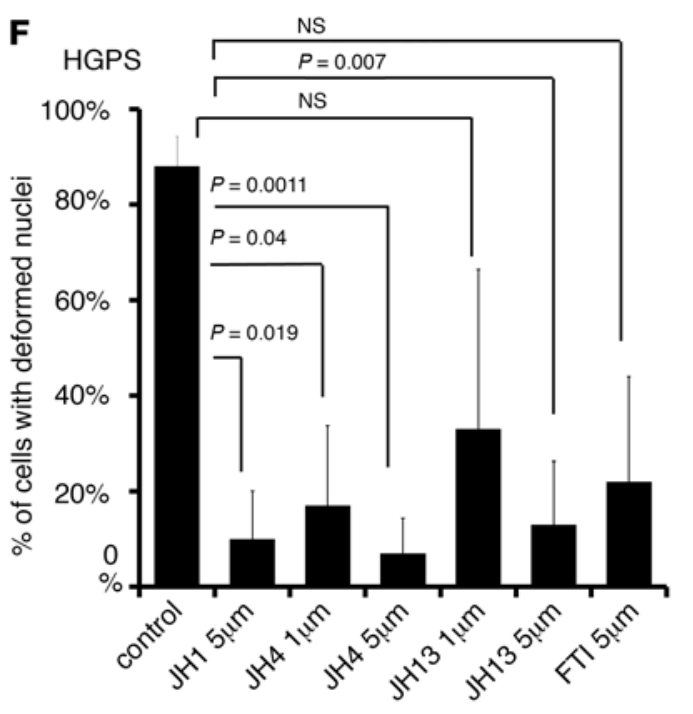


Figure 2. Ameliorating effect of JH chemicals on nuclear deformation. (A) Specific effect of JH chemicals on the binding of lamin A-progerin but not on that of lamin A-lamin A. GST pull-down assay using lysates from HEK293 cells transfected with GFP-LMNA after incubation with GSTLMNA or CST-progerin under treatment with the indicated chemicals. (B) Dissociation of lamin A and progerin by JH4 in human cells. Dissociation of progerin from lamin A/C was confirmed by IP in HEK293 cells. For this, GFP-progerin-transfected HEK293 cells were incubated with the indicated chemicals $(5 \mu \mathrm{M})$ for 24 hours. A reduction of p16 $16^{\text {INKAA }}$ was also detected in whole-cell lysate. Actin was used as a loading control. SUP, supernatent. (C) JH4 blocked the colocalization of lamin A and progerin. For this, HEK293 cells were cotransfected with GFP-LMNA and nontagged progerin (NT progerin) for 24 hours and incubated with JH4 for an additional 24 hours. $\mathrm{JH} 4$ could abolish the colocalization of lamin A and progerin (red) in the nuclear membrane or in nuclear speckles. DAPI was used for DNA staining. Scale bar: $10 \mu \mathrm{m}$. (D) JH4 blocks the interaction of lamin A and progerin in HGPS cells. Treatment with $5 \mu \mathrm{M} \mathrm{JH4}$, but not FTI-277, for 24 hours blocked the interaction of lamin A and progerin in HGPS cells. Cell lysates were immunoprecipitated with a lamin A-specific Ab (H-102, sc-20680) and immunoblotted with a progerin-specific Ab. Normal cells (9 yr; N9) were used as a negative control for progerin. (E) Effect of JH chemicals on nuclear deformation of HGPS cells. Three kinds of HGPS cells were incubated with the indicated chemicals for 24 hours and fixed for lamin $A / C$ immunofluorescence analysis (green). The same effect was observed in other HGPS cells (Supplemental Figure 4F). Scale bars: $10 \mu \mathrm{m}$. (F) Quantification of nuclear abnormalities in HGPS cells. JH chemicals, in particular JH4, ameliorated nuclear deformation of HGPS cells. $P$ values were determined by Student's $t$ test. PPT, protein pellet.

ure 3A and Supplemental Figure 5I). Then, we checked the location of biotinylated JH4 using avidin-HRP and found that JH4 was located in the nuclear membrane of progerin-transfected cells (Figure 3B) and HGPS, but not in the nuclear membrane of normal cells (Figure 3C and Supplemental Figure 5J). In addition, a biotin-avidin bead pull-down assay showed specific interaction of JH4-biotin with progerin (Figure 3D) but not with lamin A, lamin $\mathrm{B}, \mathrm{p} 53$, or emerin (Figure 3, D and E), indicating that progerin is the primary and direct target of $\mathrm{JH} 4$.

Antisenescence effect of JH chemicals on HGPS cells. We next examined the effect of $\mathrm{JH}$ chemicals on senescence. Since an important feature of senescence is an irreversible cell proliferation arrest (27), we checked the effect of JH chemicals on cell proliferation and found that these compounds (and in particular JH4) promoted the proliferation of HGPS cells (Figure 4A). In addition, JH4 promoted the proliferation of other kinds of HGPS cells (Figure 4B). Indeed, JH4 could induce the expression of Ki-67, a cell proliferation marker, in HGPS cells (Figure 4C). SA- $\beta$-gal staining, a standard senescence assay $(28,29)$, also showed antisenescent activity of JH4 in HGPS cells, which was similar to that induced by FTI treatment (Figure 4D and Supplemental Figure 6, A and B). Decoy Receptor 2 (DcR2) expression (29) was also reduced in HGPS cells at both the RNA and protein levels after treatment of these cells with JH chemicals (Supplemental Figure 6, C and D). Since progerin levels were reduced by JH4 (Supplemental Figure 6D) without a reduction of the mRNA levels (Supplemental Figure $4 \mathrm{~F}$ ), we speculated that progerin, dissociated from lamin A, may lose its stability. Indeed, JH4 could reduce the half-life of progerin (Figure 4E), which was restored by incubation with the proteasome inhibitor $N$-acetyl-leucinyl-leucinyl-norleucinal (ALLN) (Figure $4 \mathrm{~F}$ ). This result is consistent with our previous finding that
$\mathrm{JH} 4$ reduces progerin expression in immunofluorescence staining (Figure 2C). To confirm the antisenescence effect, we examined the expression of $\mathrm{H} 3 \mathrm{~K} 9 \mathrm{Me} 3$ and DNA-dependent protein kinase (DNA-PK), whose reduced levels have been proposed as markers of senescence $(9,15)$. Interestingly, JH4 and JH13 increased the expression of H3K9Me3 (Figure 4G and Supplemental Figure 6, $\mathrm{E}$ and $\mathrm{F}$ ) and the DNA-PK catalytic subunit (DNA-PKcs) in HGPS cells (Supplemental Figure 6, G and H).

Antisenescence effect of $\mathrm{JH}$ chemicals on normal aged human fibroblasts. Since progerin-induced nuclear deformation is also observed in aged cells, we hypothesized that the JH chemicals may also decelerate the normal aging process. To address this, we checked the expression of progerin in normal fibroblasts obtained from an 81-year-old donor (N81). These cells expressed progerin at the transcriptional level (Supplemental Figure 7A) and showed abnormal nuclear morphology, similar to what was observed in HGPS cells (Supplemental Figure 7B; arrowheads). These results, together with previous reports showing that progerin accumulates in aged cells and causes nuclear deformation $(8,9)$, prompted us to hypothesize that the $\mathrm{JH}$ chemicals could prevent senescence of aged cells. Indeed, the nuclear morphology of the 81-year-old cells was clearly improved by JH chemicals and FTI-277 (Figure 5A and Supplemental Figure 7C). Moreover, the slow proliferation of normal 81-year-old fibroblasts was completely rescued by JH4 (Figure 5B). To check the possible long-term toxicity of this treatment, we incubated fibroblasts from young, old, and HGPS human tissues with JH4 for 10 days and found that this compound could promote cell growth and colony formation without obvious toxicity (Supplemental Figure 7, D and E). In SA- $\beta$-gal assays, we also observed that JH4 could block cellular senescence (Figure $5 \mathrm{C}$ ). Consistently, expression of Ki-67 (30), a cell proliferation marker, was increased after JH4 treatment (Supplemental Figure 7F). Additionally, increased H3K9Me3 (Figure 5D) and reduced DcR2 and $\mathrm{p} 16^{\mathrm{INK} 4 \mathrm{~A}}$ levels were detected by JH4 treatment in normal aged fibroblasts (Figure 5E). Thus, we next checked the effect of JH4 on oncogene-induced or growth factor-induced senescence $(31,32)$. Senescence induced by IGF-1 treatment or transfection of oncogenic Ras into WI-38 (normal foreskin fibroblast) was not blocked by JH4 (Supplemental Figure 7, G and H), because oncogeneinduced senescence did not induce progerin (Supplemental Figure 7I), nor was induction of p16 blocked by JH4 treatment (Supplemental Figure 7I). In addition, JH4 did not promote cell viability in human cancer cell lines, despite a 72-hour treatment (Supplemental Figure 8A), suggesting that the antisenescence effects of $\mathrm{JH} 4$ do not result in the promotion of cancer progression. Instead, JH4 could ameliorate the nuclear deformation in renal cell carcinoma induced by progerin expression (Supplemental Figure 8B), with slight growth suppression at a high concentration (Supplemental Figure $8 \mathrm{C}$ ). These results suggest that JH chemicals, in particular JH4, selectively block progerin-mediated senescence, without growth promotion of progerin-negative cells.

Restoration of aging-related gene expression profile by JH4 treatment. To gain further insight into the biological effects of $\mathrm{JH} 4$, we investigated its impact on gene expression profiles. For this purpose, we hybridized oligonucleotide microarrays with RNA samples from HGPS-1, JH4-treated HGPS, young (9-year-old; N9), and old (81-year-old; N81) fibroblasts. Among 14,400 genes 
A

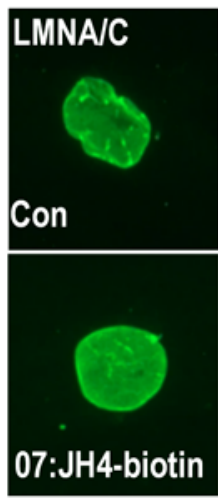

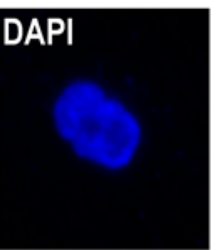

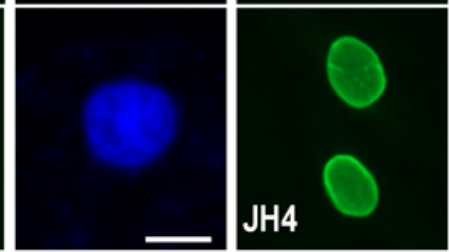

HGPS

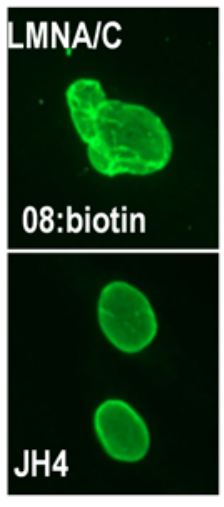

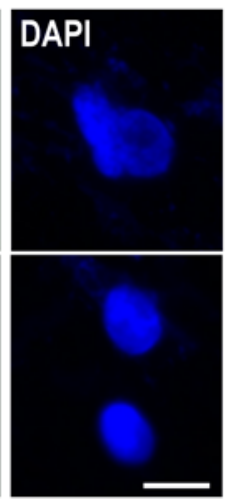

B

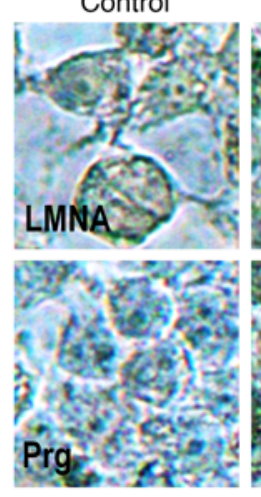

07: JH4-biotin

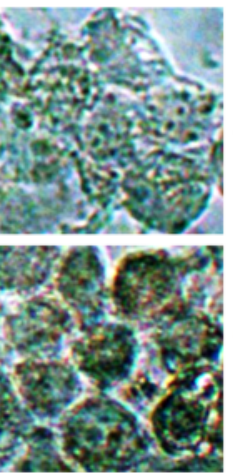

08: biotin

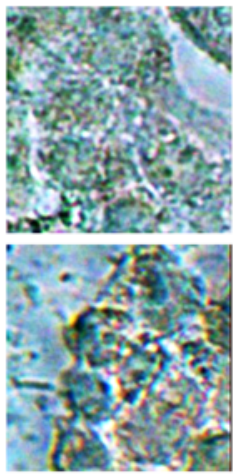

C

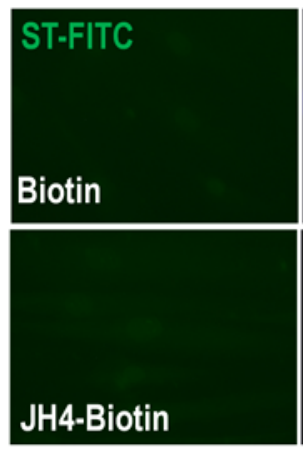

Normal (N9)

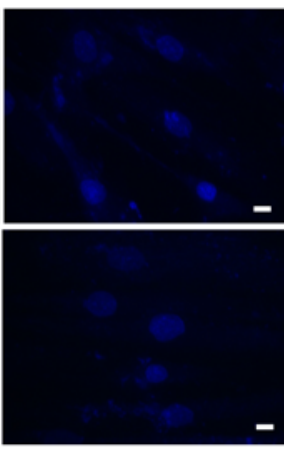

Biotin-PPT

$\mathbf{E}$
HGPS

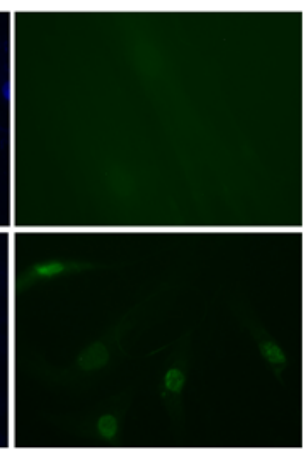

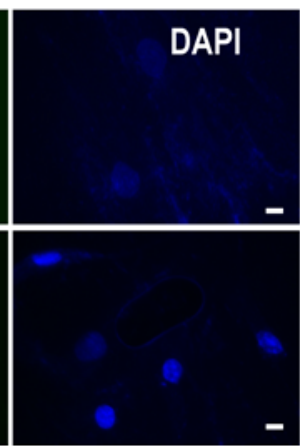

WCL
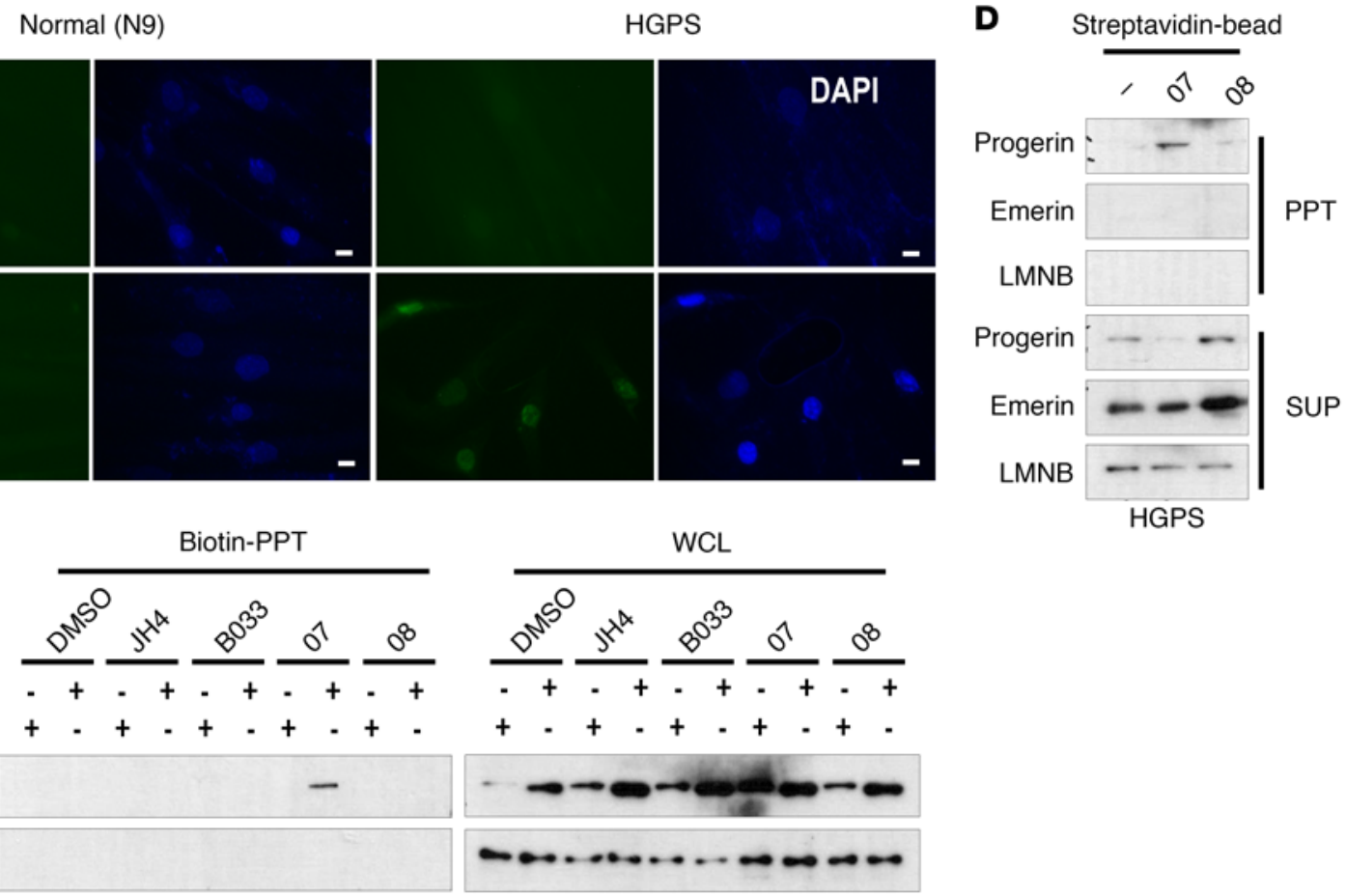

GFP-progerin

GFP-LMNA
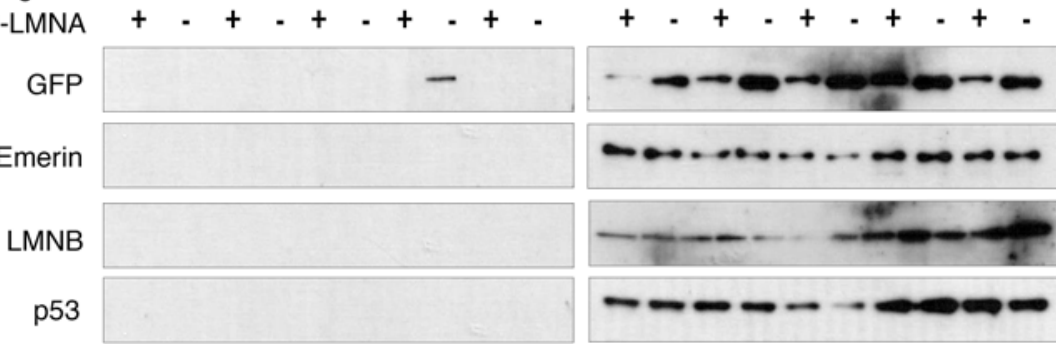

Figure 3. Progerin is a direct target of the JH4 chemical. (A) Effect of biotinylated JH4 on nuclear deformation of HGPS cells. Cells were incubated with the indicated chemicals for 24 hours and fixed for lamin A/C immunofluorescence analysis (green). Scale bars: $10 \mu \mathrm{m}$. (B) Localization of JH4 in progerin-transfected HEK293 cells. JH4-biotin (07), but not biotin alone (08), was stained in the nuclear membrane by streptavidin-HRP in progerin-transfected cells. Prg, progerin. Original magnification, $\times 200$. (C) $\mathrm{JH} 4$ locates in the nuclear membrane. After treatment with biotinylated JH4 for 48 hours, cells were fixed and stained with streptavidin-FITC. Green signal was detected only in JH4-treated HGPS. Scale bars: $10 \mu \mathrm{m}$. (D) Specific interaction of JH4 and progerin. Streptavidin-biotin-binding assay using lysates from HGPS cells incubated with biotinylated JH4 or biotin. After incubation with streptavidin-coated magnetic beads, the biotinylated JH4 with the protein complex was isolated. (E) Direct and specific interaction between JH4-biotin and progerin. Cells incubated with the indicated chemicals were lysed with RIPA and incubated with streptavidin-coated beads. Material precipitated by the biotin-streptavidin-bead complex was analyzed by Western blot analysis. GFP-progerin was precipitated by the biotin-streptavidin complex in JH4-biotin-treated cells. However, other nuclear membrane proteins such as lamin B (LMNB), emerin and GFP-lamin A, and p53 were not associated with JH4-biotin. WCL, whole-cell lysate.

present in the array, 1,921 showed differential expression (cutoff of 2-fold; Figure 5A and Supplemental Figure 9A). After elimination of nonsignificant changes resulting from the comparison between HGPS and young cells (Supplemental Figure 9A and Supplemental Table 1), we classified differential genes in an HGPS unique gene set (HUG), an aging unique gene set (AUG), and an HGPS/ aging-related gene set (HARG) (Figure 6A, Supplemental Figure 9B, and Supplemental Tables 2 and 3). Next, we defined a set of genes whose expression is restored by JH4 treatment (HARG/ JR) (298 genes; Supplemental Figure 9B and Supplemental Table 
A

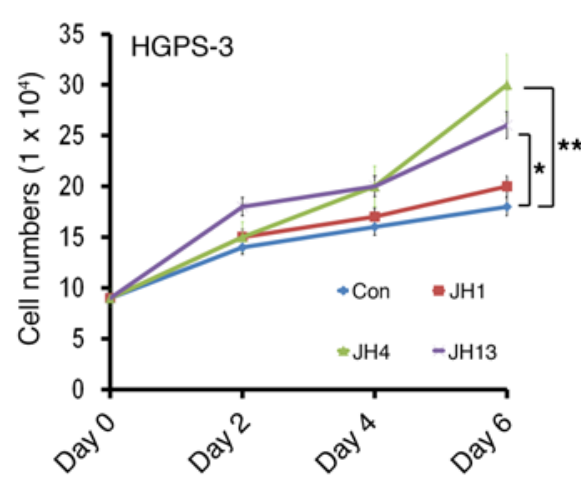

B

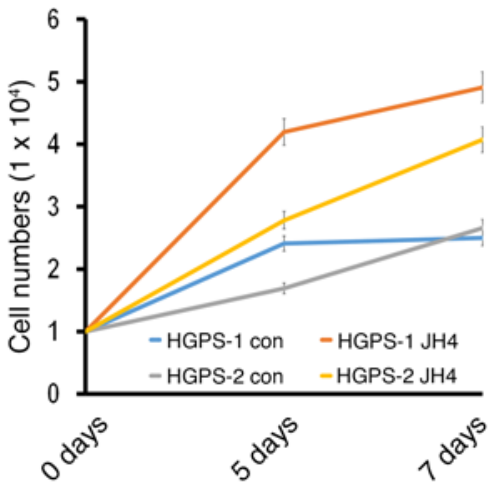

D

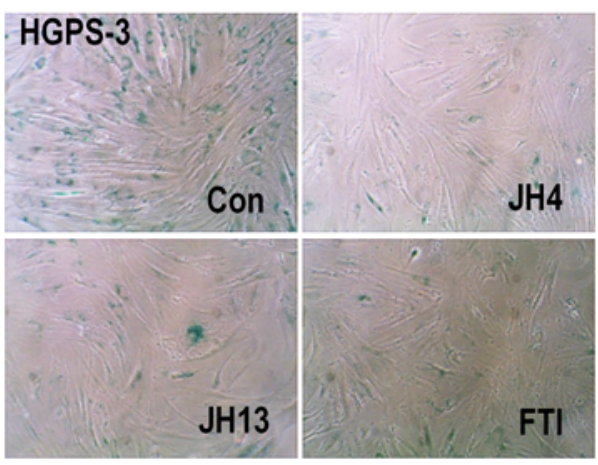

C
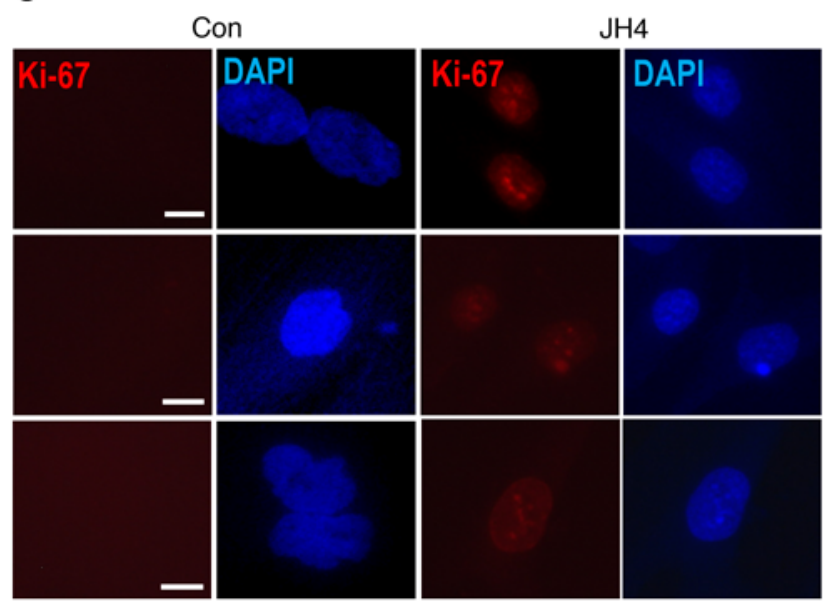

E

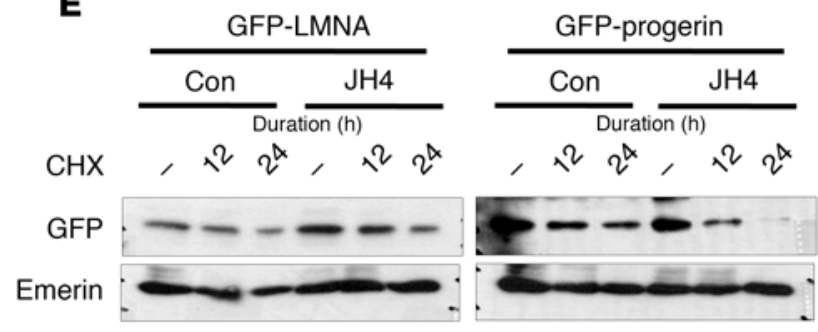

$\mathbf{F}$

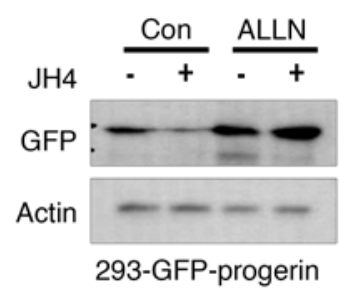

G

Н3К9Mе3
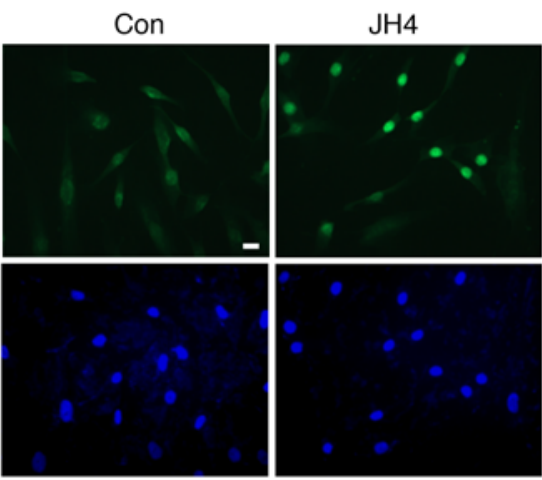

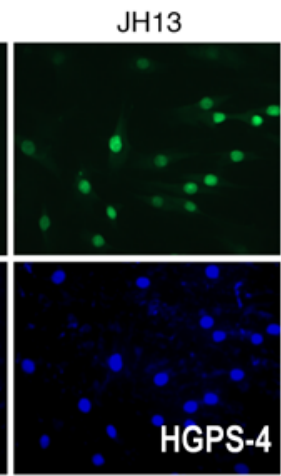

Figure 4. Antisenescence effect of the JH chemicals on HGPS cells. (A) JH chemical overcame the growth suppression of HCPS cells. After seeding at $1 \times 10^{4}$ cells per well, cell proliferation was determined by cell counting every 48 hours for 6 days. The experiment was performed in triplicate. ${ }^{*} P=0.01$, ${ }^{* *} P=0.016$. $P$ values were determined by Student's $t$ test. (B) Cell proliferation assay of normal cells obtained from a 9-year-old person (N9) under the same condition as above. JH4 could induce proliferation of normal cells, particularly at late time points (between 4 and 6 days). (C) Induction of Ki-67 by JH4 in HGPS cells. Three types of HGPS cells were incubated with JH4 for 48 hours and stained with Ki-67 Ab (red). Scale bars: $10 \mu \mathrm{m}$. (D) JH chemicals suppressed SA- $\beta$-gal expression in HGPS cells. SA- $\beta$-gal assay of HGPS cells incubated with JH chemicals ( $5 \mu \mathrm{M}$, for 48 hours). The same result was obtained for other HCPS cells (Supplemental Figure 7A). Original magnification, $\times 40$. (E) JH4 suppressed the half-life of progerin. Pulse-chase analysis using the de novo synthesis inhibitor cycloheximide (CHX) showed a rapid reduction of progerin by treatment with JH4. Cells, transfected with GFP-LMNA or GFP-progerin vectors, were incubated with $10 \mu \mathrm{M} \mathrm{CHX}$ for the indicated durations, with or without $5 \mu \mathrm{M} \mathrm{JH4}$. (F) The proteasome inhibitor ALLN blocked progerin downregulation by JH4. (G) JH chemicals induced H3K9Me3 in HGPS cells. Cells, incubated with the indicated chemicals $(5 \mu \mathrm{M})$ for 48 hours, were fixed with $100 \% \mathrm{Me}-\mathrm{OH}$ and stained with anti-H3K9Me9 Ab (green). DAPI was used for DNA staining (blue). Scale bars: $10 \mu \mathrm{m}$. Con, control.

4). To verify these transcriptomic results, we confirmed that the expression of several genes such as IL33, breast cancer 1 (BRCA1), bloom syndrome RecQ-like helicase (BLM), RAD51 recombinase (RAD51), IL6, IL8, and tumor necrosis factor superfamily member
18 (TNFSF18) was altered at both the protein and mRNA levels after JH4 treatment (Figure 6, B and C). By contrast, farnesyltransferase inhibitor (FTI) treatment did not revert the expression changes of the HARG/JR genes (Supplemental Figure 9C), indicating that the 
A

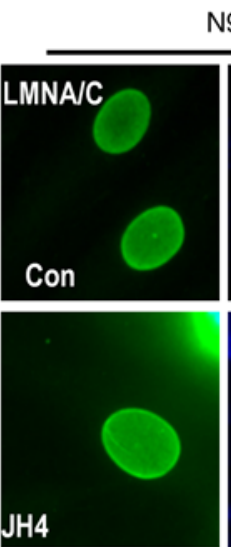

N9
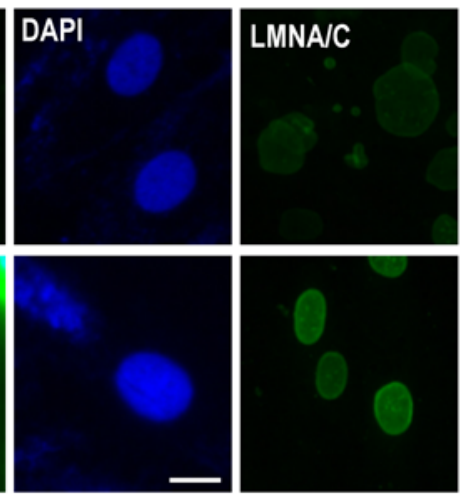

N81

C

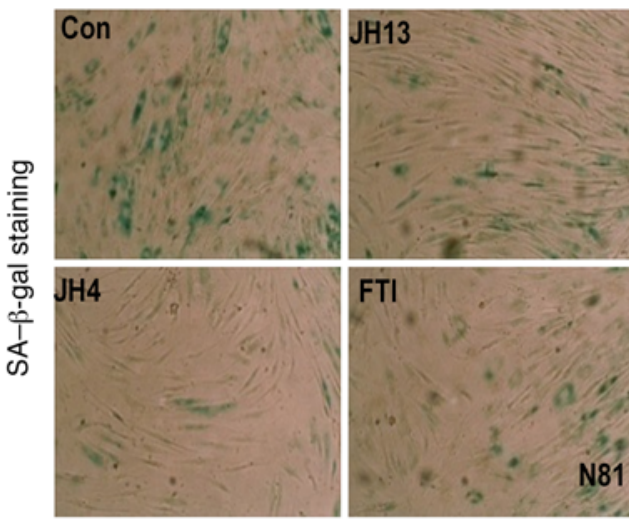

E

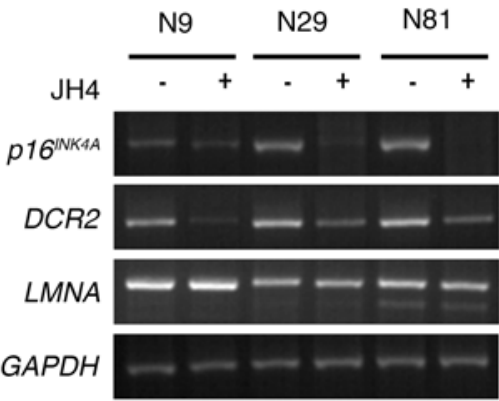

D
B
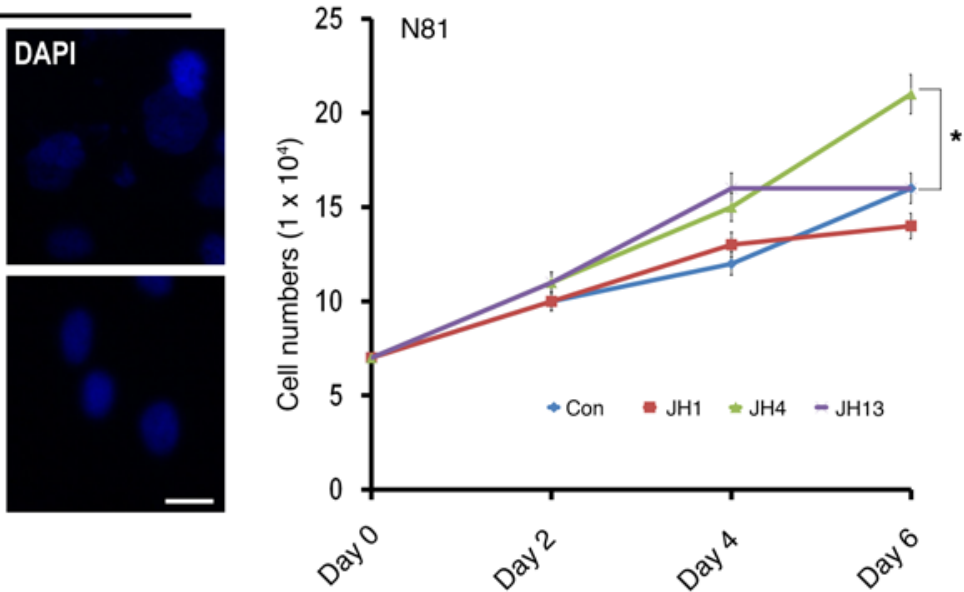

N9
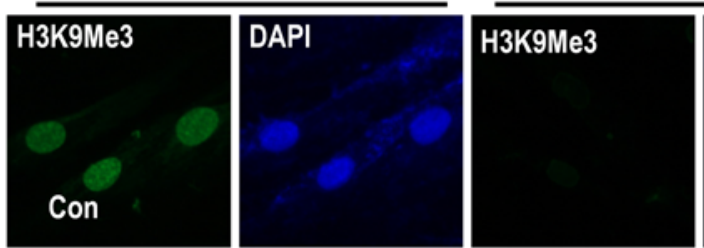

N81
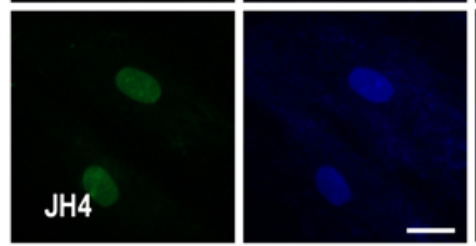

DAPI
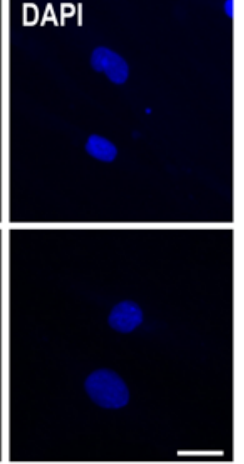

Figure 5. Antisenescence effect of JH chemicals on normal aged cells. (A) Nuclear deformation of aged fibroblasts was ameliorated by JH4. Normal cells obtained from 9-year-old (N9) and 81-year-old (N81) subjects were incubated with JH4 (5 $\mu$ M) for 48 hours and stained with anti-lamin A/C Ab (green). DAPI was used for DNA staining. Scale bars: $10 \mu \mathrm{m}$. (B) $\mathrm{JH} 4$ could induce cell proliferation in aged fibroblasts. After seeding $7.5 \times 10^{4}$ cells per well, cells were maintained for a maximum of 6 days with the indicated $\mathrm{JH}$ chemicals $(5 \mu \mathrm{M})$. Cell numbers were calculated every 48 hours. The slow proliferation of cells from N81 compared with that of cells from N9 (Figure 4B) was completely rescued by JH4 treatment. ${ }^{*} P=0.013$. The $P$ value was determined by Student's $t$ test for analysis of statistical significance between 2 groups. (C) JH4 suppressed senescence of N81 fibroblasts. An SA- $\beta$-gal assay was performed on cells from N81 after treatment with the indicated chemicals $(5 \mu \mathrm{M})$ for 48 hours. Original magnification, $\times 40$. (D) JH4 induced H3K9Me3 expression in normal aged cells. Immunofluorescence for H3K9Me3 (green) was performed in N9 and N81 cells under treatment with the indicated chemicals ( $5 \mu \mathrm{M})$ for 48 hours. Scale bars: $10 \mu \mathrm{m}$. (E) JH4 suppressed DCR2 and p16 INK4A in normal human cells. Reverse transcriptase-PCR (RT-PCR) analysis of the indicated genes was performed in normal cell lines (N9, N29, and N81) after treatment with JH4 for 48 hours.

effects of JH4 and FTI are markedly different. Since the alternation of gene expression is due to a different genetic background but not to aging, we examined the expression of several genes induced or suppressed in aged fibroblasts and HGPS cells in other kinds of human aged fibroblasts and different HGPS cells. Expression levels of IL33, RAD51, and BRCA1 were reduced in 3 kinds of aged fibro- blasts (N81; N72, 72-year-old; and N94, 94-year-old) and HGPS-2 cells compared with the levels detected in fibroblasts from N9 (Supplemental Figure 9D). In contrast, $I L 6$ and $I L 8$ were overexpressed in these cells (Supplemental Figure 9D). This result suggested that alternation of these expression levels would result from aging. To investigate the biological significance of these transcriptional alter 
A

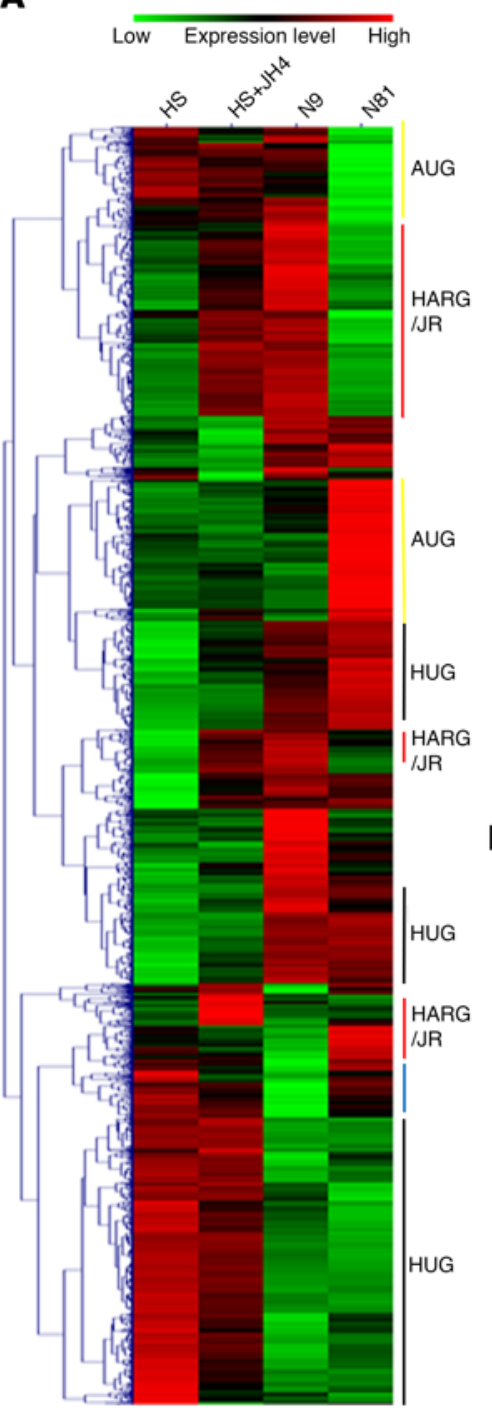

B

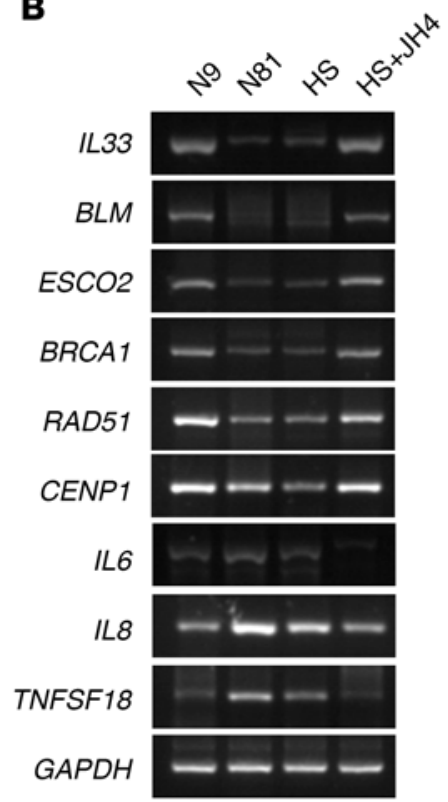

C

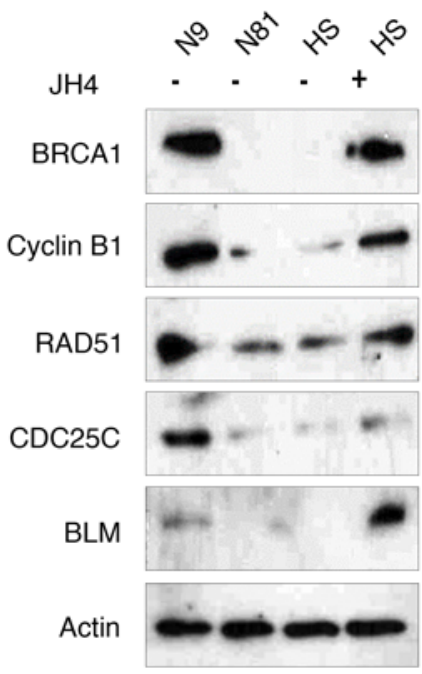

E
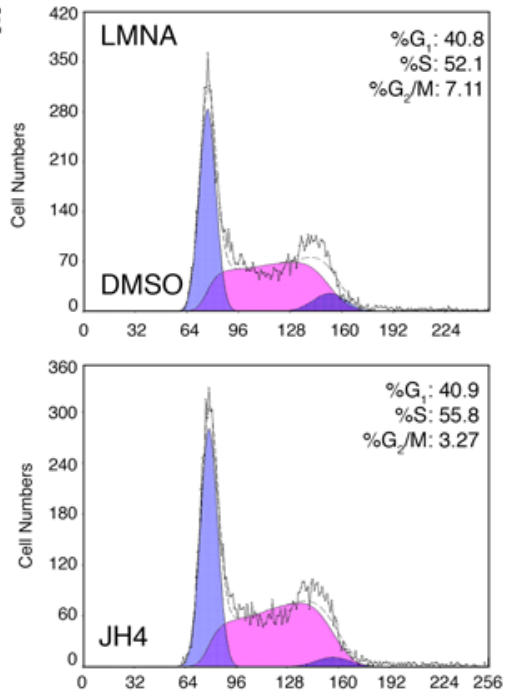
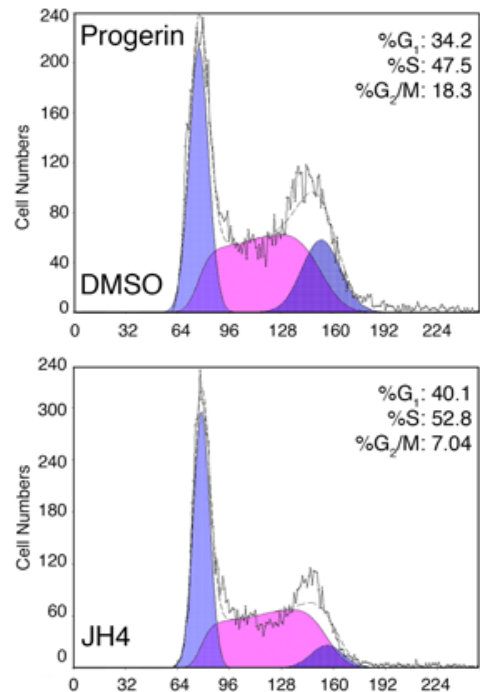

D

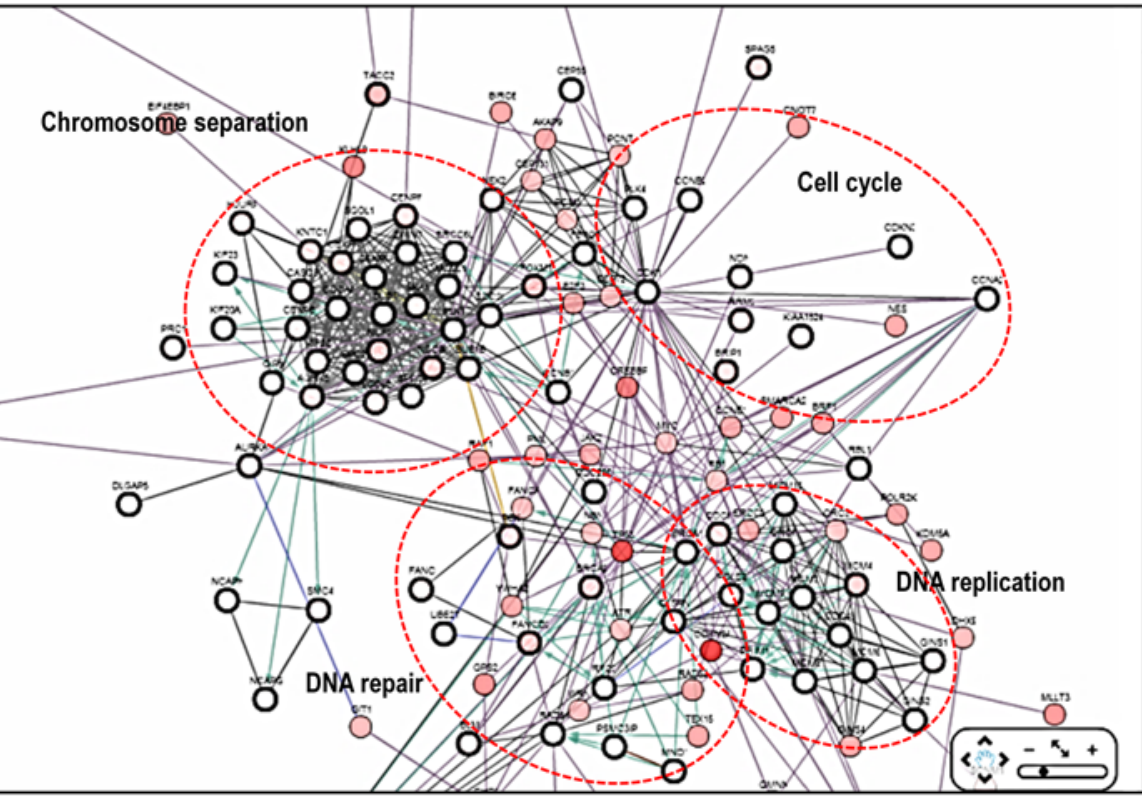

Figure 6. Aging-related gene expression is recovered by JH4 treatment. (A) Heatmap of gene expression profiles showing a difference of at least 2-fold in both cell lines. Colors represent values normalized to the range of intensity for each gene in all conditions. (B) Reduced expression of IL33, BRCA1, BLM, and RAD51 in HGPS cells was reverted by JH4. Conversely, IL6, IL8, and TNFSF18 overexpression was suppressed by JH4 treatment. (C) JH4 could alter HGPS-specific gene expression. RAD51, BRCA1, cyclin B1, and CDC25C protein expression levels were increased by treatment with JH4. (D) Network analysis using cBioportal. Cenes in the HARG/JH group were clustered into chromosome separation, DNA replication, DNA repair, and cell-cycle groups. (E) JH4 overcame progerin-induced cell-cycle arrest. Cell-cycle arrest at the $G_{2} / M$ phase by progerin transfection was released by $\mathrm{JH} 4$ treatment ( $5 \mu \mathrm{M}$, for 24 hours). 
A

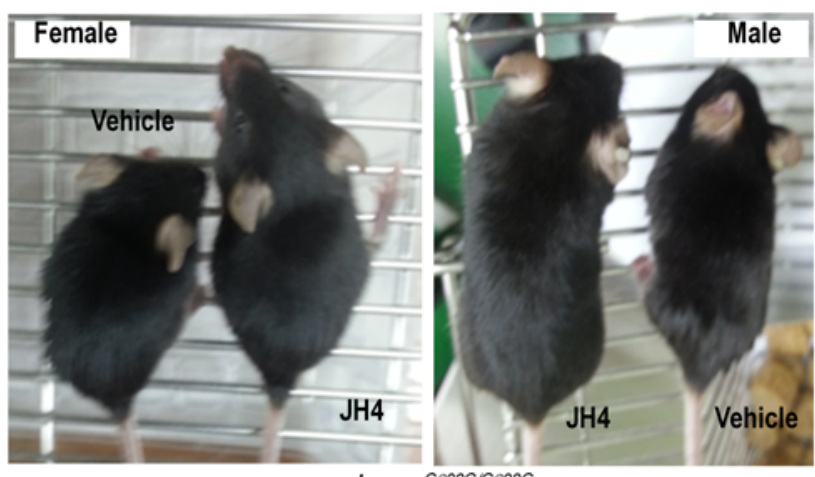

Lmna ${ }^{G 609 G / G 609 G}$

C 807 Grip strength (male)

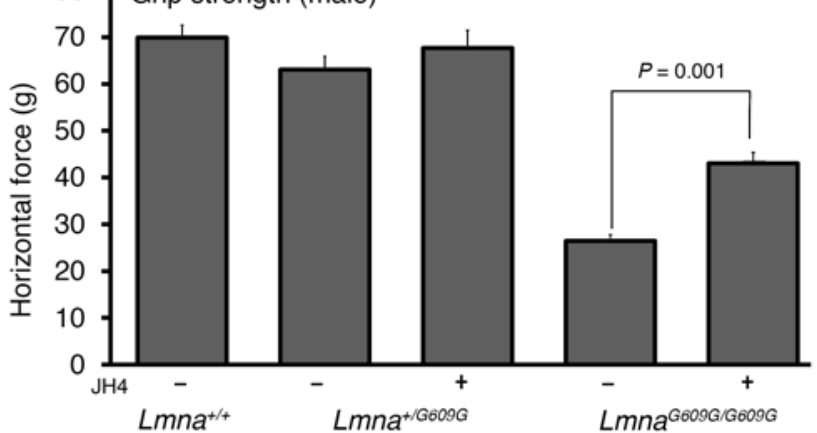

E

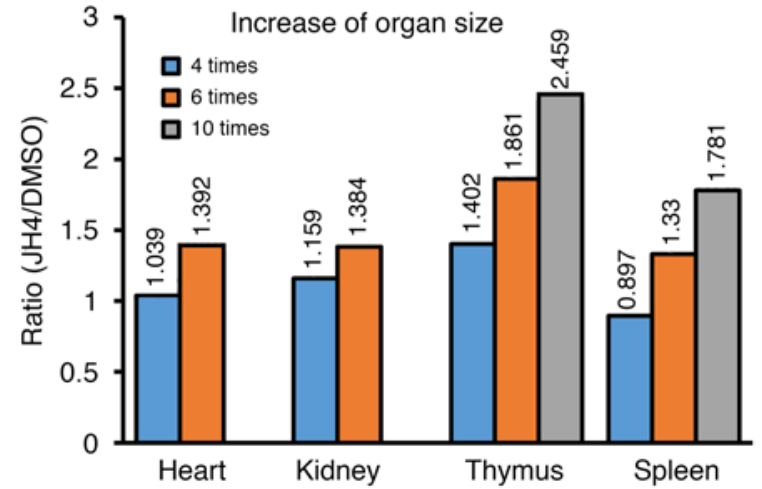

G

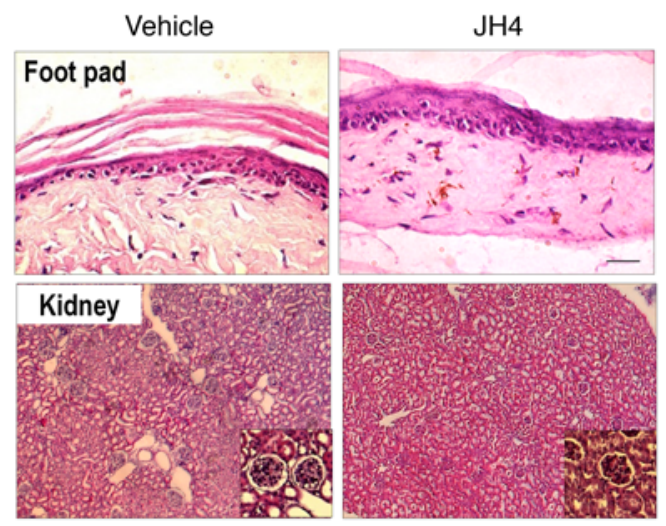

Lmna $a^{60096 / 60096}$

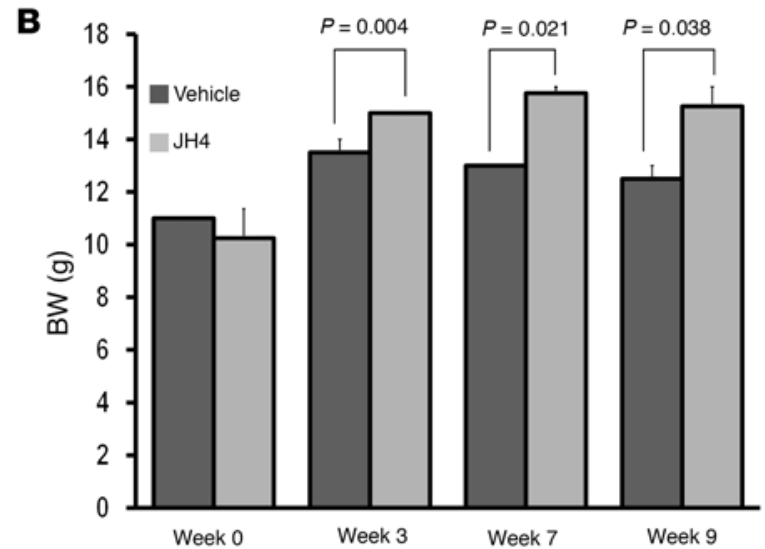

D
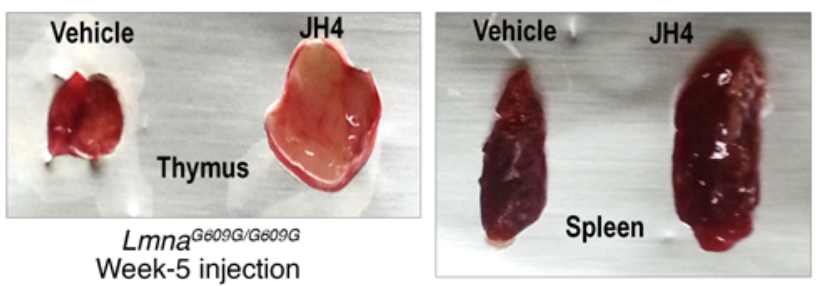

$\mathbf{F}$
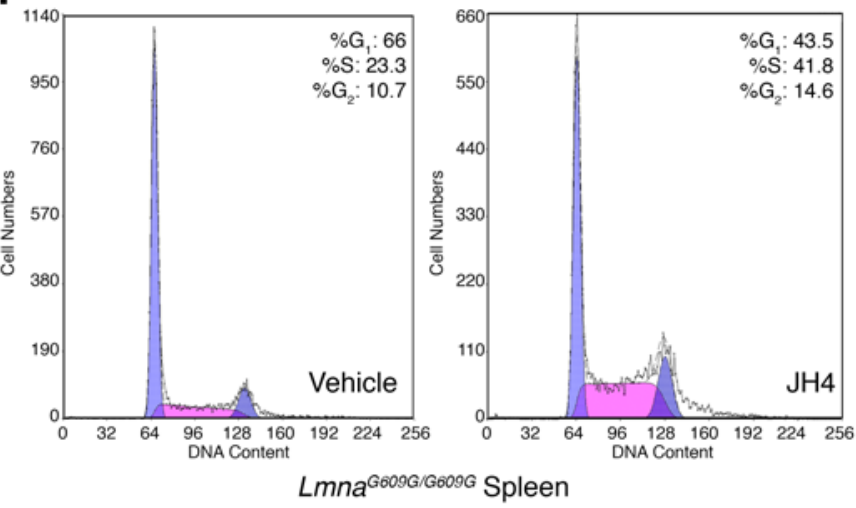

Lmna ${ }^{\text {G009G/G609G }}$ Spleen

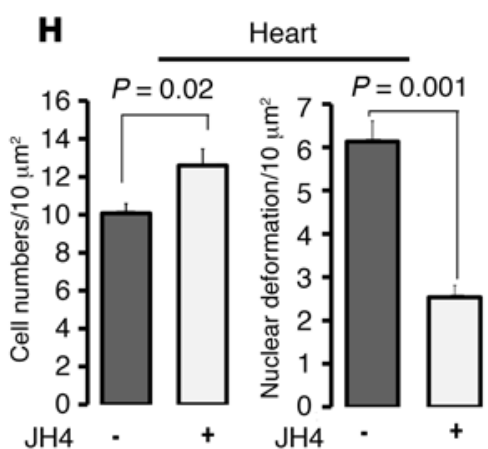

I Vehicle

$\mathrm{JH} 4$

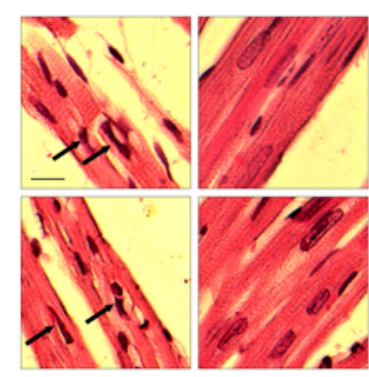

J

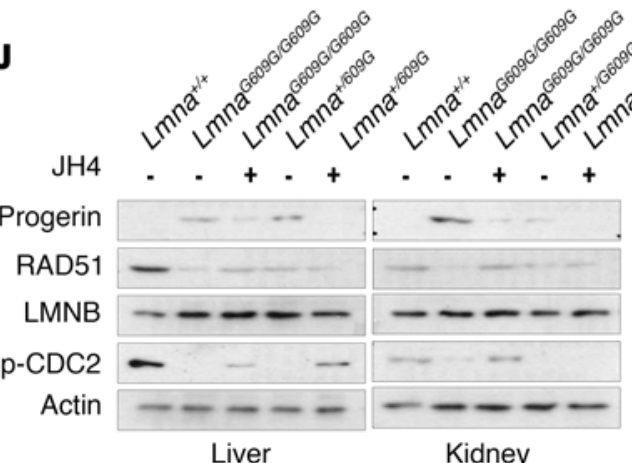


Figure 7. JH4 prevents progeroid phenotypes in Lmna ${ }^{6609 C / 6609 C}$ mice. (A) Representative photographs of 8-week-old $L m n a^{66096 / 6609 C}$ mice (female, left; male, right) treated with $\mathrm{JH} 4$ (10 mg/kg twice per week). Mice were injected 8 times (from 4 weeks of age). (B) Increase in BW of $L m n a^{66096 / 6609 C}$ mice following JH4 injection. $P$ values were determined by Student's $t$ test. (C) Increase in muscle strength following JH4 injection. Grip strength was measured for $L m n a^{C 6096 / 6609 C}, L m n a^{+/ 6609 C}, L m n a^{+/+}$male mice by pulling the spring balance after treatment with $\mathrm{JH} 4$ or vehicle alone. $P$ value was determined by Student's $t$ test. (D) Growth morphology of thymus and spleen from 9-week-old $L m n a^{66096 / 6609 C}$ mice treated with vehicle or JH4. An obvious enlargement of both organs after JH4 treatment was observed. (E) JH4 injection time-dependent organ size increase. Compared with vehicleinjected mice, the size of several organs was gradually increased by treatment time. Numbers above each bar indicate the fold increase at each time point (see also Supplemental Figure 14). (F) JH4 injection promoted the cell cycle of splenocytes obtained from 7-week-old mice (injected 6 times). Cell-cycle promotion was observed in JH4-treated cells. (C) Rescue of cell and tissue defects. Foot pad skin was thickened and dermal connective tissue was enriched in JH4-treated animals (upper panels). Kidney tissue was well organized after $\mathrm{J} 4$ treatment. Tissues were obtained from 18-week-old (vehicle) and 22-week-old (JH4) mice. Scale bar: $80 \mu \mathrm{m}$, original magnification $\times 100$. $(\mathbf{H})$ Increase in cell density and reduction of deformed cell nuclei in JH4-treated heart tissue obtained from the same mice above. On the basis of the histology, nuclear morphology (right) and cell density (left) were counted for 3 different mice and 5 different regions per mouse by 3 researchers. $P$ values were determined by Student's $t$ test. (I) Representative images of heart muscle. Arrows indicate deformed nuclei. Scale bar: $10 \mu \mathrm{m}$. (J) JH4 suppressed progerin and induced RAD51 and phosphorylated CDC2 ( $p-C D C 2)$. Western blot analysis was performed using extracts from liver and kidney tissue obtained from 18 -week-old sibling mice.

ations, we analyzed the altered gene networks using cBioportal $(33,34)$ and found that many genes in the HARG/JR group were clustered in 4 functional categories: chromosome segregation, cell cycle, DNA repair, and DNA replication (Figure 6D). For example, genes encoding BUB1 (budding uninhibited by benzimidazoles 1), shugoshin-like 1 (SGOL1), centromere protein (CENP) family proteins (chromosome segregation), cyclin B2, cyclin-dependent kinase 1 (CDK1) (cell cycle), BRCA1, BRCA2, RAD51 (DNA repair), and minichromosome maintenance protein complex (MCM) family proteins were listed in this group (Supplemental Figure 9, E and F). On the basis of gene function, we grouped all JH4-responding genes into 18 categories (Supplemental Figure 9G). Consistent with what we observed with the protein network, the majority of HARG/ JR was clustered in the cell cycle, DNA replication, chromosome regulation, and DNA repair. In agreement with these findings, JH4 could overcome the progerin-induced cell-cycle arrest, in particular at the $\mathrm{G}_{2} / \mathrm{M}$ phase (Figure $6 \mathrm{E}$ ).

JH4 blocks progerin-induced lamin A aggregation in ER during the $G_{2} / M$ phase. Because many of the JH4-responsive genes shown in Supplemental Table 4 and Figure 4B are involved in cell-cycle and chromosome regulation and progerin induces cell-cycle arrest at the $\mathrm{G}_{2} / \mathrm{M}$ checkpoint (Figure $6 \mathrm{E}$ ), we examined the effect of JH4 on the behavior of lamin A at the $\mathrm{G}_{2} / \mathrm{M}$ phase. Dispersal of lamin A upon treatment with Taxol (paclitaxel) was observed in young, but not aged, cells. Rather, lamin A surrounded chromosomal or nuclear DNA (Supplemental Figure 10A, white arrows). However, treatment with JH4 could promote lamin A elimination in aged cells (Supplemental Figure 10A, yellow arrows). We also observed that progerin-induced lamin A aggregation in nocodazol- or Taxol-treated cells was abolished by JH4, but not by FTI-277 (Supplemental Figure 10, B and C). To gain further insight into this observation, we analyzed the localization of progerin in nocodazol- or Taxoltreated cells. Cytoplasmic aggregates in nocodazol- or Taxoltreated cells were undetectable upon $\mathrm{JH} 4$ treatment, but not after incubation with FTI-277 (Supplemental Figure 11A). Given the role of the ER in nuclear lamina remodeling during the $G_{2} / M$ phase (23), we investigated the subcellular localization of lamin A and an ER-specific marker (35) in JH4-treated cells and found that aggregates of lamin $\mathrm{A}$ in the ER disappeared after JH4 treatment (Supplemental Figure 11B). Next, we checked the location of progerin after arrest at the $\mathrm{M}$ phase by Taxol treatment and found that JH4 also blocked ER-associated progerin during the $\mathrm{M}$ phase (Supplemental Figure 11C). To confirm whether M-phase aggregates contain lamin A-progerin complexes, we costained lamin A-GFP and progerin in Taxol-treated cells. Colocalized lamin A and progerin in the non-nuclear region was abrogated by $\mathrm{JH} 4$ (Supplemental Figure 11D). This result indicates that progerin disrupts lamin A dissemination and reduction during the $\mathrm{G}_{2} / \mathrm{M}$ phase by direct binding to this nuclear envelope protein. Indeed, it has been recently reported that HGPS cells show downregulated and defective poly(ADP-ribose) polymerase 1 (PARP1) in $\mathrm{G}_{2} / \mathrm{M}$-phase progression (36).

Therapeutic effect of JH4 on the HGPS mouse model. To evaluate the therapeutic potential of JH4 in vivo, we injected i.p. $10 \mathrm{mg} / \mathrm{kg}$ of this compound twice per week into 4-week-old Lmna ${ }^{\mathrm{G} 6096 / 6609 \mathrm{G}}$ HGPS mice, a suitable murine model of human HGPS (37). After 4 weeks of treatment, we observed a significant increase in BW (Figure 7, A and B), which continuously increased out to 12 weeks (Supplemental Figure 12A). By contrast, vehicle-treated mice showed a significant decrease in BW after the eighth week (Figure 7B and Supplemental Figure 12A). At 11 to 12 weeks, the difference in BW between vehicle- and JH4-treated mice reached $4 \mathrm{~g}$ (about 25\%; Supplemental Figure 12A). In addition, injection of JH4 increased grab strength (Figure 7C) and suppressed hair loss at week 15 (Supplemental Figure 12B). Consistently with the increase in BW, the size of different organs was increased by JH4 administration (Figure 7D). Moreover, organ size recovery was fully dependent on the number of injections given (Figure 7E and Supplemental Figure 13), such that 5 weeks after the start of treatment (10 injections), thymus and spleen volumes were enlarged by approximately 2-fold when compared with tissues from vehicle-treated mice (Figure 7E). Since progerin induces a cell-cycle arrest that seems to be critical for growth retardation $(17,18,37)$, we also analyzed cellcycle dynamics and found that it was also promoted by JH4 (Figure $7 \mathrm{~F}$ and Supplemental Figure 12C) in a number of injectiondependent ways (Supplemental Figure 13, A7 and B7). Histological analysis revealed that $\mathrm{JH} 4$ increased skin thickness, reduced kidney destruction, and alleviated nuclear deformation (Figure $7 \mathrm{G}$ and Supplemental Figure 12D). To obtain more detailed evidence, we measured the cell density and frequency of deformed nuclei in heart (Figure 7, H and I), liver (Supplemental Figure 12E), lung (Supplemental Figure 12F), and kidney (Supplemental Figure 12G). In all examined tissues, JH4 treatment increased cell density and reduced the number of deformed nuclei. For example, the reduction in cell numbers in the same region or in tubes and 
A

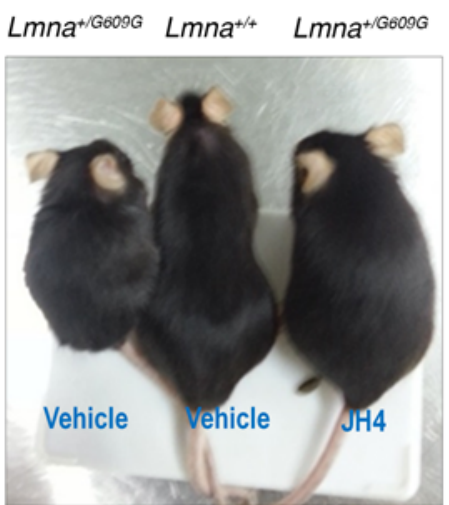

C
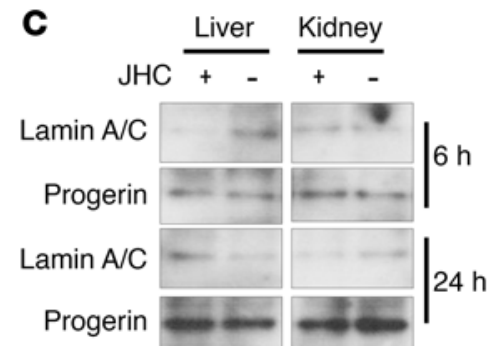

IP: Progerin

E

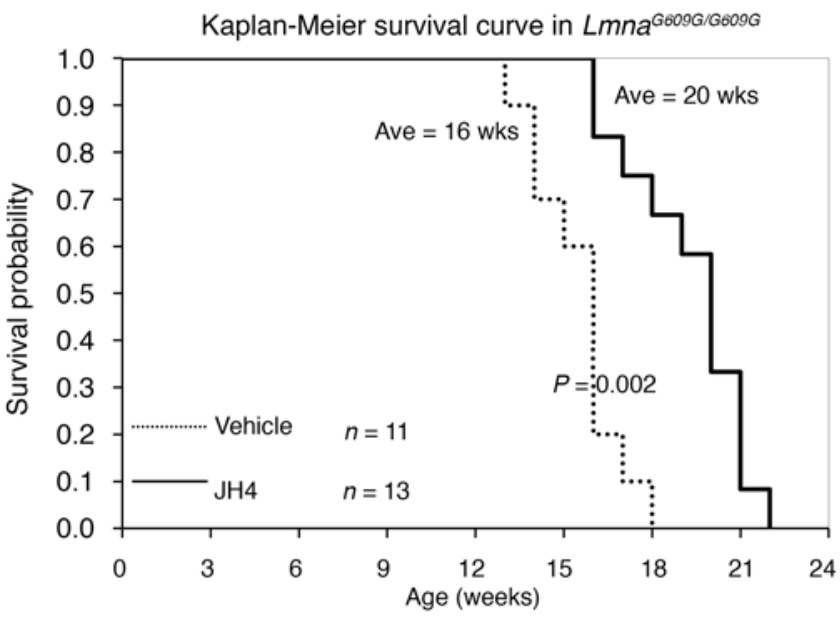

G

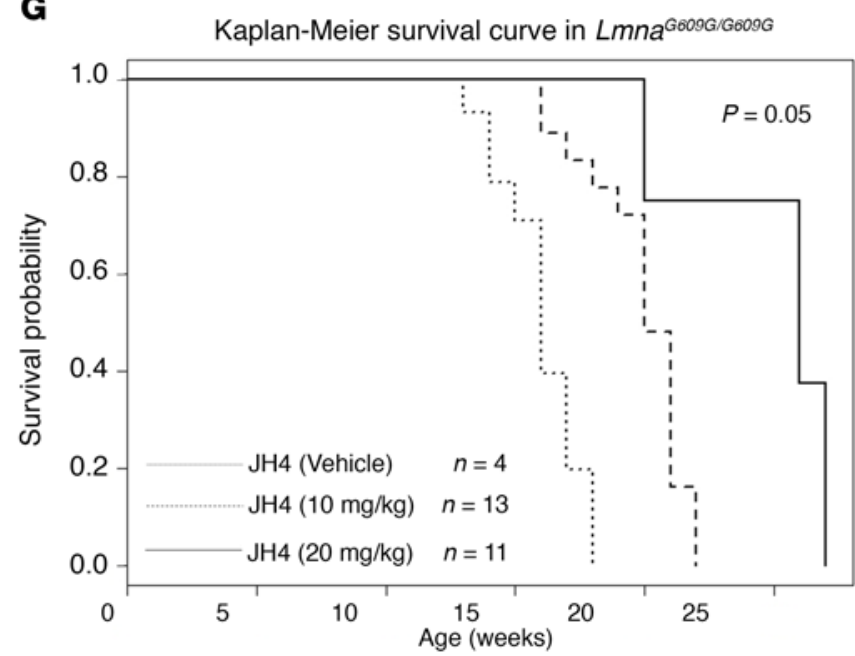

B

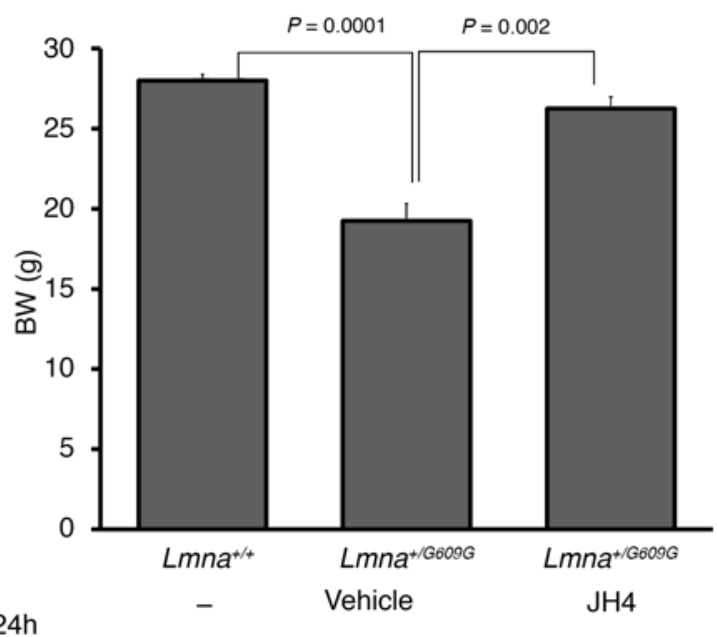

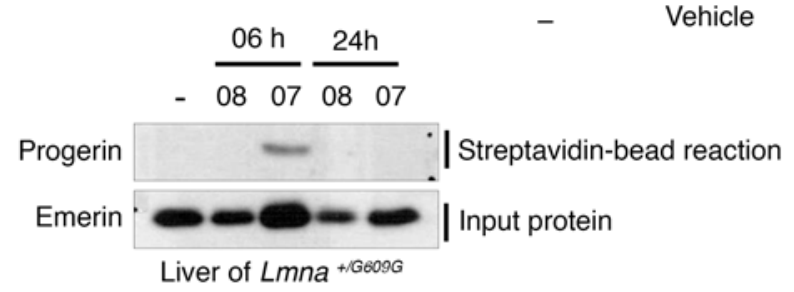

$\mathbf{F}$

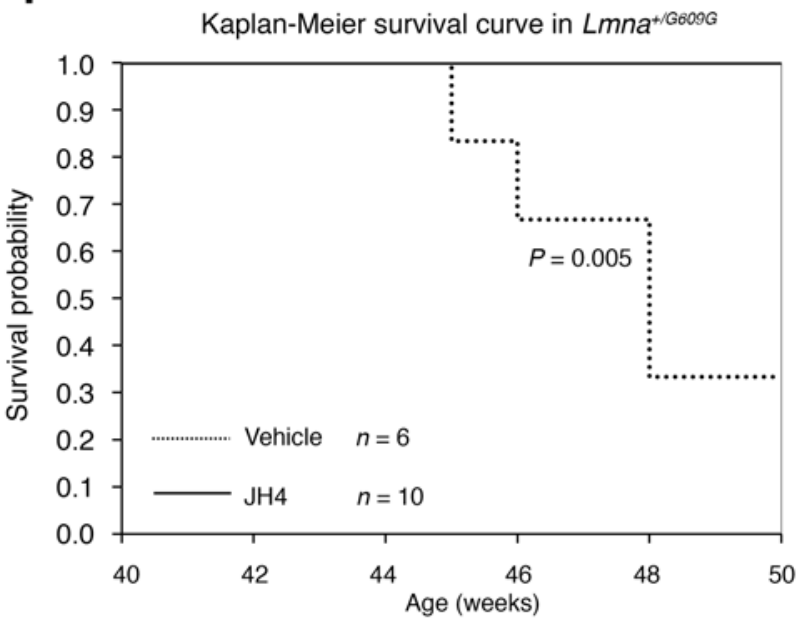


Figure 8. Lifespan extension in progerin-expressed mice. (A) Representative photograph of 40 -week-old $\mathrm{Lmna}^{+/ C 609 \mathrm{C}}$ control or JH4-treated mice. (B) BW of 40-week-old $\mathrm{Lmna}^{+/ / 609 C}$ control and JH4-treated mice. (C) In vivo effect of $\mathrm{JH} 4$ on binding between progerin and lamin A/C. Lmna ${ }^{+/ 6609 C}$ mice that received a single injection of JH4 $(10 \mathrm{mg} / \mathrm{kg})$ were sacrificed for IP analysis of liver and kidney. After 6 hours, JH4 could disrupt the interaction of progerin and lamin A. After 24 hours, dissociation of progerin and lamin A was detected in kidney. Extracted proteins were immunoprecipitated with progerin $A b$, and the precipitated proteins were subjected to Western blotting with anti-lamin $A / C$ or anti-progerin $A b$. (D) Detection of JH4 in liver. Biotin-JH4 was injected into $L m n a^{+/ C 609 C}$ mice, and liver lysates were reacted with streptavidin beads. Precipitated proteins were analyzed by Western blotting. (E) Kaplan-Meier survival plots for $L m n a^{C 6096 / 6609 C}$ mice treated with JH4 $(n=13)$ or with vehicle alone $(n=11)$. (F) Kaplan-Meier survival plots for $L m n a^{+/ 6609 G}$ mice treated with vehicle $(n=6)$ or JH4 $(n=10)$. (C) Extension of lifespan by high-dose injection of JH4. A dose of $20 \mathrm{mg} /$ $\mathrm{kg} \mathrm{JH} 4$ could extend lifespan by as much as 26 weeks. (B and E-C) $P$ values were determined by Student's $t$ test.

early cysts in Lmna ${ }^{\mathrm{G6096/6609G}}$ mice was recovered following JH4 treatment and returned to numbers similar to those detected in WT mice (Supplemental Figure 12I). To examine the effect of JH4 at the molecular level, we measured the expression of progerin as well as array-target genes such as Rad51 and cell-cycle regulators. Consistent with our microarray data described above, the expression level of these genes was restored by JH4 treatment in an injection time-dependent manner (Figure 7J, Supplemental Figure 13, A8 and B8). Moreover, progerin expression was reduced to undetectable levels (Figure 7J).

Specific effect of JH4 on progerin-expressing mice. Because $L m n a^{+/ G 609 G}$ progerin heterozygous mice also exhibit progeroid features, we checked the effect of JH4 on these animals and found that injection of JH4 (twice/week) over a 20-week period also ameliorated a number of aging phenotypes present in these mice, such as loss of BW (Figure 8, A and B, and Supplemental Figure 14A) and dermal and heart muscle alterations (Supplemental Figure 14B). Moreover, treatment of $\mathrm{Lmna}^{+/ G 609 G}$ mice with JH4 could result in recovery of their BW to that of normal mice (Figure 8B). To investigate whether the in vivo antiprogeria effects of $\mathrm{JH} 4$ are due to the inhibition of progerin-lamin A binding or to an unknown side effect of this compound, we decided to test JH4 in a progerin-independent progeria model, namely the zinc metallopeptidase STE24-deficient mouse (Zmpste24 $4^{-/}$) (38), in which the progeroid phenotype is caused by prelamin A rather than by progerin accumulation. Despite the nearly identical progeroid phenotype of Zmpste24 $4^{-/}$and $L m n a^{\mathrm{G} 6096 / 6009 G}$ mice, JH4 did not produce any noticeable change of growth in Zmpste24 $4^{--}$mice (Supplemental Figure $14 \mathrm{C}$ ), demonstrating that the antiaccelerated aging activity of $\mathrm{JH} 4$ is directly related to its inhibition of progerin's deleterious effects. Indeed, the interaction between progerin and lamin $\mathrm{A} / \mathrm{C}$ in tissues was disrupted by a single $\mathrm{JH} 4$ injection (Figure $8 \mathrm{C}$ ), following its metabolic tract. Disruption of progerin-lamin A/C binding by JH4 in liver at 6 hours was observed after 24 hours in kidney. We were able to recover the progerin-binding biotin-JH4 (labeled "07" in Figure 8D) in liver 6 hours after injection, indicating, as we expected, that JH4 could work in an in vivo system.

JH4 extends lifespan in the progerin mouse model. Next, we assessed the lifespan of $L m n a^{\mathrm{G} 6096 / G 609 G}$ progeroid mice after JH4 injection. As shown in Figure 8E, the lifespan of JH4-treated progeroid mice was extended by more than 4 weeks compared with that of vehicle-treated mice. Although JH4 treatment did not fully overcome the short lifespan of these animals, considering the low concentration $(10 \mathrm{mg} / \mathrm{kg})$ and frequency (2 times/week) of administration, it appears to have a promising effect. In fact, JH4

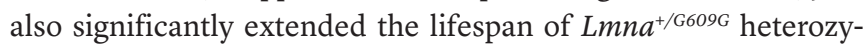
gous mice (Figure 8F). Since biological effect of JH4 was dosedependent, we injected $20 \mathrm{mg} / \mathrm{kg} \mathrm{JH} 4$ into $\mathrm{Lmna}^{\mathrm{G} 6096 / \mathrm{G} 609 \mathrm{G}}$ progeroid mice. From this experiment, we found that $20 \mathrm{mg} / \mathrm{kg} \mathrm{JH} 4$ could extend lifespan by as much as 26 weeks (Figure 8G). These results suggest that if $\mathrm{JH} 4$ can be retained at a higher concentration, lifespan could be more dramatically extended and that JH4 would be a strong therapeutic drug candidate for the treatment of HGPS.

\section{Discussion}

The aging process is complicated, and a full understanding of the molecular mechanisms controlling it is not likely to be realized in the near future. However, some basic clues are known from studying human progeria syndromes such as HGPS (39-41). Although HGPS is caused by progerin, a product of a point mutation in the LMNA gene, progerin is also expressed in normal aged cells as well as in cancers (14). Thus, understanding the pathological function of progerin is useful for the development of a therapeutic strategy to treat HGPS as well as for the prevention of aging.

In fact, over the past decade, the farnesylation of progerin has been targeted for drug development to treat HGPS. $(24,42)$. Because the farnesylated domain of progerin rigidly anchors it to the nuclear membrane, it may induce nuclear deformation. Blocking farnesylation by FTIs in HGPS cells restores nuclear morphology, cell proliferation, and heterochromatin organization. Moreover, treatment with FTIs in HGPS-like mouse models improves lifespan, growth, and BW and relieves premature aging symptoms such as bone defects (23). In particular, FTIs can extend the lifespan of Zmpste24 $4^{--}$mice (24). However, a clinical trial of FTI did not produce promising results (43), indicating that other targets should be explored.

In this study, we found that the most important target of progerin is lamin $\mathrm{A} / \mathrm{C}$. Indeed, progerin shows strong binding affinity for lamin A/C but not lamin B (Figure 1E). On this basis, we found new chemicals that can block the interaction between progerin and lamin $\mathrm{A} / \mathrm{C}$ through direct interaction with progerin (Figure 2B and Figure 3E). Since the chemical JH4 can bind selectively to progerin, there would be very minimal, nonspecific side effects. In our study, JH4 did not show an effect on the progerin-independent progeria model (Zmpste24 ${ }^{-/}$mice; Supplemental Figure 14C) or on mouse and monkey cell lines (data not shown). These results indicate that $\mathrm{JH} 4$ has potential use as a progerin-inhibitory drug.

Although JH4 did not fully overcome the short lifespan of the $L m n a^{\mathrm{G} 6096 / 6009 G}$ mouse model, given the low dose and infrequent injections of the compound, an optimized treatment strategy using JH4 could offer an effective therapy. Indeed, the same scheduled injection produced more obvious effects in $\mathrm{Lmna}^{+/ 6609 \mathrm{G}}$ mice (Figure 8), and JH4 appeared to be excreted within 24 hours.

In summary, in this study, we have identified 3 compounds, $\mathrm{JH} 1, \mathrm{JH} 4$, and JH13, that efficiently block progerin-lamin A/C 
binding and reverse or alleviate different senescence features characteristic of HGPS cells, including growth arrest, SA- $\beta$-gal accumulation, and nuclear aberrations. Moreover, we observed similarly favorable effects of $\mathrm{JH}$ chemicals on normal aged fibroblasts, which also express progerin. Indeed, JH4 suppressed SA- $\beta$-gal activity and increased the proliferation of these cells. Moreover, many genes altered by JH4 treatment were common between HGPS and aging cells. The fact that this compound also rescued several progeroid features of $L m n a^{\mathrm{G609G/G609G}}$ mice and extended the lifespan of this HGPS mouse model represents a proof of principle for the in vivo relevance of blocking progerin-lamin A/C interaction as a new treatment strategy for HGPS and, eventually, for age-associated alterations involving nuclear envelope abnormalities.

\section{Methods}

Animal experiments. Progerin-heterozygous Lmna ${ }^{+/ G 609 G}$ mice were provided by Carlos López-Otín (Universidad de Oviedo, Asturias, Oviedo, Spain). Timed matings of heterozygous $L m n a^{+/ G 609 G}$ and homozygous Lmna ${ }^{G 609 G / G 609 G}$ males and females were conducted, and chemical was intraperitoneally injected for indicated weeks or months (Figure 8, E-G). All mice were maintained on outbred C57BL6/J backgrounds.

Histology. Following sacrifice at the end of the injection regimen, the indicated organs (foot pad, kidney, and heart) were isolated in PBS and fixed in $10 \%$ formaldehyde in PBS at $4^{\circ} \mathrm{C}$ for 6 to 12 hours. Tissue was dehydrated using ethanol (50\%, 70\%, 90\%, $3 \times 100 \%$; 5 minutes each) and paraffin embedded for sectioning. Sections (5- $\mu$ m-thick) were used for H\&E staining.

Chemical screening and chemical synthesis. For chemical screening, an ELISA was established by modifying a previously described platform (44). Details on the synthetic methods used are provided in the Supplemental material.

Cell culture and reagents. Human fibroblast cells were obtained from the Coriell Cell Repositories. Human cancer cell lines (HCT116, A549, and HEK293), obtained from ATCC, were maintained in liquid medium containing $10 \% \mathrm{FBS}$ and $1 \%$ antibiotics at $37^{\circ} \mathrm{C}$. Nonhuman cell lines such as Vero (monkey), HICIC7 (mouse), MDCK (canine), and $\mathrm{Mv1Lu}$ (mink) were obtained from the Korean Cell Line Bank (KCLB, Seoul, Korea).

Plasmids and transfection. GFP-progerin and GFP-fused lamin A expression vectors were provided by T. Misteli (National Cancer Institute [NCI], Bethesda, Maryland, USA). GFP-lamin B1 and lamin C were provided by J. Lammerding (Brigham and Women's Hospital/ Harvard Medical School, Boston, Massachusetts, USA). Mutant forms of lamin A were gifts of F. Tesson (University of Ottawa, Ottawa, Ontario, Canada). The Myc-ARF vector and OST-LAMINA $\triangle 50$ vector were purchased from Addgene. Transfection was performed using jetPEI (Polyplus Transfection) following the manufacturer's protocol.

Recombinant proteins and GST pull-down assay. For investigation of protein-protein interactions, recombinant proteins were generated. Recombinant lamin A N-terminal region (His-LMNA-N; aa residues 1-300) and middle region (His-LMNA-M; 301-564 aa) was cloned into His-tagged pPROEXHT. GST-fused recombinant lamin A-C terminal region (GST-LMNA) and progerin C-terminal region (GST-progerin) were produced by PCR cloning 100 aa upstream of the termination codon through PCR. Full-length $144^{\mathrm{ARF}}$ was produced by PCR, cloned into the pGEX vector, and confirmed by DNA sequencing. These recombinant proteins were purified using a nickel column. For the binding assay, GST-bead-lamin A/C or progerin-C was incubated with lysate from 293 cells that had been transfected with recombinant lamin A N-terminal region, lamin A middle region, GFP-lamin $\mathrm{A}$, lamin $\mathrm{B}$ or lamin $\mathrm{C}$ for 2 hours at room temperature. After washing twice with PBS and once with RIPA buffer $(50 \mathrm{mM}$ Tris, $150 \mathrm{mM} \mathrm{NaCl}$, $1 \%$ NP-40, 0.5\% deoxycholate, and 0.5\% SDS), precipitated materials were collected and subjected to SDS-PAGE and Western blotting using anti-GFP, His, and GST Abs.

Streptavidin-biotin-binding assay. Biotin-conjugated JH4 was produced by chemical synthesis (see the Chemical Synthesis section in the Supplemental materials). To determine the location of JH4-biotin, cells were incubated with biotin or biotin-JH4 for the indicated durations (Figure 3) and fixed with 2\% PFA. After permeabilization, blocking with BSA, and prereaction with $\mathrm{H}_{2} \mathrm{O}_{2}$ to eliminate endogenous HRP activity, cells were incubated with streptavidin-HRP (SigmaAldrich) and DAB solution (Vector Laboratories). To investigate the direct binding of JH4 and progerin, cells lysates, precleared by streptavidin-bead, were incubated with biotin-JH4 or biotin and streptavidin-bead, sequentially. The precipitated materials were subjected to SDS-PAGE and Western blot analysis.

Microarray data. The full data set is available in the NCBI's Gene Expression Omnibus (GEO) database (GEO GSE84147; www.ncbi. nlm.gov/geo/query/acc.cgi acc=GSE84147).

Statistics. All data are expressed as the mean \pm SEM. Statistical significance was determined using a 2-tailed Student's $t$ test. For all experiments, a $P$ value of less than 0.05 was considered statistically significant.

Study approval. All in vivo studies were performed at an Association for Assessment and Accreditation of Laboratory Animal Carecertified facility, in compliance with animal policies approved by Pusan National University (Busan, Korea) and the Universidad de Oviedo (Asturias, Oveido, Spain).

\section{Author contributions}

SJL, YSJ, MHY, SK, AYO, JHL, SYJ, TGW, HYC, SKK, KJC, HYL, KL, GK, MKN, NCH, CB, JMPF, and BJP performed the experiments. SJL, YSJ, and BJP conceived the experimental designs. CLO, GYS, and BJP wrote the manuscript. SJL, GYS, and BJP analyzed the data.

\section{Acknowledgments}

This research was supported by the Basic Science Research Program through the National Research Foundation of Korea (NRF), funded by the Ministry of Science, ICT and Future Planning (NRF-2013R1A1A2010008, to BJP; 2009-0093815, to GYS; 2014R1A2A2A01005455, to KL; and NRF-2013R1A1A2063077, to SJL); the Progeria Research Foundation; the Ministerio de Economía y Competitividad of Spain; and the Red Temática de investigación cooperativa en cáncer-Instituto de Salud. The Instituto Universitario de Oncología is supported by Obra Social Cajastur-Asturias. CLO is an Investigator of the Botin Foundation.

Address correspondence to: Bum-Joon Park, 609-735, Busandaehak-ro 63, Geumjeong-gu, Busan, South Korea. Phone: 82.51.510.2220; E-mail: bjpark1219@pusan.ac.kr. Or to: Gyu Yong Song, 34134. Deahak-ro 99, Yuseong-gu, Daejeon, South Korea. Phone: 82.42.821.5926; E-mail: gysong@cnu.ac.kr. 
1. Gordon LB, Rothman FG, López-Otín C, Misteli T. Progeria: a paradigm for translational medicine. Cell. 2014;156(3):400-407.

2. Burke B, Stewart CL. Life at the edge: the nuclear envelope and human disease. Nat Rev Mol Cell Biol. 2002;3(8):575-585.

3. Kipling D, Davis T, Ostler EL, Faragher RG. What can progeroid syndromes tell us about human aging? Science. 2004;305(5689):1426-1431.

4. Miller RA. 'Accelerated aging': a primrose path to insight? Aging Cell. 2004;3(2):47-51.

5. López-Otín C, Blasco MA, Partridge L, Serrano M, Kroemer G. The hallmarks of aging. Cell. 2013;153(6):1194-1217.

6. De Sandre-Giovannoli A, et al. Lamin a truncation in Hutchinson-Gilford progeria. Science. 2003;300(5628):2055.

7. Eriksson M, et al. Recurrent de novo point mutations in lamin A cause Hutchinson-Gilford progeria syndrome. Nature. 2003;423(6937):293-298.

8. McClintock D, et al. The mutant form of lamin A that causes Hutchinson-Gilford progeria is a biomarker of cellular aging in human skin. PLoS One. 2007;2(12):e1269.

9. Scaffidi P, Misteli T. Lamin A-dependent nuclear defects in human aging. Science. 2006; 312(5776):1059-1063.

10. Scaffidi P, Misteli T. Reversal of the cellular phenotype in the premature aging disease Hutchinson-Gilford progeria syndrome. Nat Med. 2005;11(4):440-445.

11. Goldman RD, et al. Accumulation of mutant lamin $A$ causes progressive changes in nuclear architecture in Hutchinson-Gilford progeria syndrome. Proc Natl Acad Sci U S A. 2004;101(24):8963-8968.

12. Huang $\mathrm{S}$, et al. Correction of cellular phenotypes of Hutchinson-Gilford Progeria cells by RNA interference. Hum Genet. 2005;118(3-4):444-450.

13. Tang Y, Chen Y, Jiang H, Nie D. Promotion of tumor development in prostate cancer by progerin. Cancer Cell Int. 2010;10:47.

14. Jung YS, et al. Loss of VHL promotes progerin expression, leading to impaired p14/ARF function and suppression of p53 activity. Cell Cycle. 2013;12(14):2277-2290.

15. Liu GH, et al. Recapitulation of premature ageing with iPSCs from Hutchinson-Gilford progeria syndrome. Nature. 2011;472(7342):221-225.

16. Zhang J, et al. A human iPSC model of Hutchinson-Gilford progeria reveals vascular smooth muscle and mesenchymal stem cell detects. Cell Stem Cell. 2011;8(1):31-45.

17. Cao K, Capell BC, Erdos MR, Djabali K, Collins
FS. A lamin A protein isoform overexpressed in Hutchinson-Gilford progeria syndrome interferes with mitosis in progeria and normal cells. Proc Natl Acad Sci U S A. 2007;104(12):4949-4954.

18. Dechat $\mathrm{T}$, et al. Alterations in mitosis and cell cycle progression caused by a mutant lamin A known to accelerate human aging. Proc Natl Acad Sci US A. 2007;104(12):4955-4960.

19. Huang S, Risques RA, Martin GM, Rabinovitch PS, Oshima J. Accelerated telomere shortening and replicative senescence in human fibroblasts overexpressing mutant and wild-type lamin A. Exp Cell Res. 2008;314(1):82-91.

20. Kubben N, et al. Identification of differential protein interactors of lamin A and progerin. Nucleus. 2010;1(6):513-525.

21. Zhong N, Radu G, Ju W, Brown WT. Novel progerininteractive partner proteins hnRNP E1, EGF, Mel 18, and UBC9 interact with lamin A/C. Biochem Biophys Res Commun. 2005;338(2):855-861.

22. Kapinos LE, et al. Characterization of the headto-tail overlap complexes formed by human lamin A, B1 and B2 "half-minilamin" dimers. JMol Biol. 2010;396(3):719-731.

23. Yang L, Guan T, Gerace L. Integral membrane proteins of the nuclear envelope are dispersed throughout the endoplasmic reticulum during mitosis. J Cell Biol. 1997;137(6):1199-1210.

24. Fong LG, et al. A protein farnesyltransferase inhibitor ameliorates disease in a mouse model of progeria. Science. 2006;311(5767):1621-1623.

25. Kamijo T, Weber JD, Zambetti G, Zindy F, Roussel MF, Sherr CJ. Functional and physical interactions of the ARF tumor suppressor with p53 and Mdm2. Proc Natl Acad Sci U S A. 1998;95(14):8292-8297.

26. Ju WN, Brown WT, Zhong N. Association of progerin-interactive partner proteins with lamina proteins: Mel18 is associated with emerin in HGPS. Beijing Da Хие Хие Bao. 2009;41(4):397-401.

27. Hayflick $L$. The limited in vitro lifetime of human diploid cell strains. Exp Cell Res. 1965;37:614-636.

28. Dimri GP, et al. A biomarker that identifies senescent human cells in culture and in aging skin in vivo. Proc Natl Acad Sci U S A. 1995;92(20):9363-9367.

29. Collado M, Serrano M. The power and the promise of oncogene-induced senescence markers. Nat Rev Cancer. 2006;6(6):472-476.

30. Schlüter C, et al. The cell proliferation-associated antigen of antibody Ki-67: a very large, ubiquitous nuclear protein with numerous repeated elements, representing a new kind of cell cycle-maintaining proteins. JCell Biol. 1993;123(3):513-522.
31. Ben-Porath I, Weinberg RA. The signals and pathways activating cellular senescence. Int $J$ Biochem Cell Biol. 2005;37(5):961-976.

32. Kim KS, et al. Induction of cellular senescence by insulin-like growth factor binding protein- 5 through a p53-dependent mechanism. Mol Biol Cell.2007;18(11):4543-4552.

33. Cerami $\mathrm{E}$, et al. The cBio cancer genomics portal: an open platform for exploring multidimensional cancer genomics data. Cancer Discov. 2012;2(5):401-404.

34. Gao J, et al. Integrative analysis of complex cancer genomics and clinical profiles using the cBioPortal. Sci Signal. 2013;6(269):pl1.

35. Ong HL, et al. Relocalization of STIM1 for activation of store-operated $\mathrm{Ca}^{2+}$ entry is determined by the depletion of subplasma membrane endoplasmic reticulum $\mathrm{Ca}^{2+}$ store. J Biol Chem. 2007;282(16):12176-12185.

36. Zhang H, Xiong Z, Cao K. Mechanisms controlling the smooth muscle cell death in progeria via down-regulation of ploy(ADPribose) polymerase 1. Proc Natl Acad Sci U S A. 2014;111(22):E2261-E2270.

37. Osorio FG, et al. Splicing-directed therapy in a new mouse model of human accelerated aging. Sci Transl Med.2011;3(106):106ra107.

38. Pendás AM, et al. Defective prelamin A processing and muscular and adipocyte alterations in Zmpste24 metalloproteinase-deficient mice. Nat Genet. 2002;31(1):94-99.

39. Burtner CR, Kennedy BK. Progeria syndromes and ageing: what is the connection?. Nat Rev Mol Cell Biol. 2010;11(8):567-578.

40. Worman HJ, Ostlund C, Wang Y. Diseases of the nuclear envelope. Cold Spring Harb Perspect Biol. 2010;2(2):a000760.

41. Kudlow BA, Kennedy BK, Monnat RJ. Werner and Hutchinson-Gilford progeria syndromes: mechanistic basis of human progeroid diseases. Nat Rev Mol Cell Biol. 2007;8(5):394-404.

42. Yang SH, et al. Blocking protein farnesyltransferase improves nuclear blebbing in mouse fibroblasts with a targeted Hutchinson-Gilford progeria syndrome mutation. Proc Natl Acad Sci US A. 2005;102(29):10291-10296.

43. Gordon LB, et al. Clinical trial of a farnesyltransferase inhibitor in children with HutchinsonGilford progeria syndrome. Proc Natl Acad Sci U S A. 2012;109(41):16666-16671.

44. Lee SH, et al. Antitumor effect of novel small chemical inhibitors of Snail-p53 binding in K-Ras-mutated cancer cells. Oncogene. 2010;29(32):4576-4587. 